ESAIM: M2AN 47 (2013) 663-688

DOI: $10.1051 / \mathrm{m} 2 \mathrm{an} / 2012041$
ESAIM: Mathematical Modelling and Numerical Analysis

www.esaim-m2an.org

\title{
ON POWER SERIES SOLUTIONS FOR THE EULER EQUATION, AND THE BEHR-NEČAS-WU INITIAL DATUM
}

\author{
Carlo Morosi ${ }^{1}$, Mario Pernici $^{2}$ and Livio Pizzocchero 3,4
}

\begin{abstract}
We consider the Euler equation for an incompressible fluid on a three dimensional torus, and the construction of its solution as a power series in time. We point out some general facts on this subject, from convergence issues for the power series to the role of symmetries of the initial datum. We then turn the attention to a paper by Behr, Nečas and Wu, ESAIM: M2AN 35 (2001) 229-238; here, the authors chose a very simple Fourier polynomial as an initial datum for the Euler equation and analyzed the power series in time for the solution, determining the first 35 terms by computer algebra. Their calculations suggested for the series a finite convergence radius $\tau_{3}$ in the $H^{3}$ Sobolev space, with $0.32<\tau_{3}<0.35$; they regarded this as an indication that the solution of the Euler equation blows up. We have repeated the calculations of E. Behr, J. Nečas and H. Wu, ESAIM: M2AN 35 (2001) 229-238, using again computer algebra; the order has been increased from 35 to 52 , using the symmetries of the initial datum to speed up computations. As for $\tau_{3}$, our results agree with the original computations of E. Behr, J. Nečas and H. Wu, ESAIM: M2AN 35 (2001) 229-238 (yielding in fact to conjecture that $0.32<\tau_{3}<0.33$ ). Moreover, our analysis supports the following conclusions: (a) The finiteness of $\tau_{3}$ is not at all an indication of a possible blow-up. (b) There is a strong indication that the solution of the Euler equation does not blow up at a time close to $\tau_{3}$. In fact, the solution is likely to exist, at least, up to a time $\theta_{3}>0.47$. (c) There is a weak indication, based on Padé analysis, that the solution might blow up at a later time.
\end{abstract}

Mathematics Subject Classification. 35Q31, 76B03, 35B44, 76M60.

Received March 30, 2012. Revised July 17, 2012.

Published online March 4, 2013.

\section{INTRODUCTION}

Let us consider the three-dimensional Euler equation for a homogeneous incompressible fluid (of unit density) with initial datum $u_{0}$, i.e.,

$$
\frac{\partial u}{\partial t}=-u \bullet \nabla u-\nabla p, \quad u(x, 0)=u_{0}(x)
$$

\footnotetext{
Keywords and phrases. Euler equation, existence and regularity theory, blow-up, symbolic computation.

1 Dipartimento di Matematica, Politecnico di Milano, P.za L. da Vinci 32, 20133 Milano, Italy. carlo.morosi@polimi.it

2 Istituto Nazionale di Fisica Nucleare, Sezione di Milano, Via Celoria 16, 20133 Milano, Italy. mario.pernici@mi.infn.it

3 Dipartimento di Matematica, Università di Milano, Via C. Saldini 50, 20133 Milano, Italy

4 Istituto Nazionale di Fisica Nucleare, Sezione di Milano, Italy. livio.pizzocchero@unimi.it
} 
The unknown is the divergence-free velocity field $(x, t) \mapsto u(x, t)$; we assume periodic boundary conditions, so $x=\left(x_{1}, x_{2}, x_{3}\right)$ ranges in the three dimensional torus $(\mathbf{R} / 2 \pi \mathbf{Z})^{3}$. In the sequel, we often write $u(t)$ for the function $x \mapsto u(x, t)$.

One can try a solution of the above Cauchy problem in the form of a power series $u(t)=\sum_{j=0}^{+\infty} u_{j} t^{j}$ (with $u_{j}=u_{j}(x)$ ); such power series have been the object of rather extensive investigations. Morf et al. [17], Frisch [13], Brachet et al. $[9,22]$, and other authors (see the bibliography of the cited references) have constructed by computer algebra techniques many terms of the power series for specific initial data, consisting of simple Fourier polynomials; more precisely, the data analyzed in these works are the so-called "Taylor-Green vortex", and other vortices proposed by Kida [16]. The cited authors have also discussed the possibility of a blow-up (i.e., finitetime divergence of $u(t)$ ) on the grounds of their computer algebra calculations. Another initial datum (again a Fourier polynomial) has been considered by Behr, Nečas and Wu [6]; these authors have constructed 35 terms of the power series, and claimed to have found evidence for a blow-up of the solution; however, in comparison with the vortices of Taylor-Green and Kida, the Behr-Nečas-Wu initial datum has received less attention in the literature.

The purpose of the present paper is twofold.

(i) First of all, we wish to point out a number of general facts on the solutions of the Euler equation and, in particular, on the convergence of the power series $\sum_{j=0}^{+\infty} u_{j} t^{j}$; this is the subject of Sections 2 and 3 . Here we report some results extracted from the existing literature on the Euler equation in spaces of analytic functions and/or in Sobolev spaces; in addition to these results, we present some remarks of ours and propose a general treatment to discuss the symmetries of the initial datum and their effects on the solution of the Euler equation. We think it is not useless to collect all these theoretical statements in a unifying framework, suitable for direct application to computer algebra calculations.

(ii) Our second aim is to reanalyze the power series for the Behr-Nečas-Wu initial datum, both from the theoretical and from the computational viewpoint; this is the subject of Sections 4, 5 and 6 . First of all we apply to the Behr-Nečas-Wu case our general setting for the symmetries of the initial datum. We calculate the symmetry subgroup of the Behr-Nečas-Wu datum (that we recognize to be the dihedral group of order 6 ; this group also determines what we call the pseudo-symmetry space of the datum).

With these premises, we present a novel computation of the power series for the Behr-Nečas-Wu datum, based on a Python program written for this purpose; this computation attains the order 52. The Python program uses an exact representation of rational numbers as ratios of integer, so as not to introduce rounding errors; furthermore, it employs the symmetries of the initial datum to reduce the amount of calculations.

The results of such computations can be analyzed using the theoretical framework of Sections 2 and 3. Our conclusions are the following:

(a) We agree with the estimates of [6], according to which the power series under consideration has a convergence radius $0.32<\tau_{3}<0.35$ in the Sobolev space $H^{3}$; in fact, our computations suggest $0.32<\tau_{3}<0.33$. However, we disagree from the authors of [6] when they interpret the finiteness of $\tau_{3}$ as indicating a blow-up of the solution.

(b) On the contrary, we give evidence that the solution $u(t)$ of the Euler equation exists for $t$ significantly larger than $\tau_{3}$. In fact, analyzing the power series for the squared Sobolev norm $\|u(t)\|_{3}^{2}$, we find a strong indication for a convergence radius $\theta_{3}$ such that $0.47<\theta_{3}<0.50$ (see our Eq. (5.31)). By a general criterion (based on the classical works of Bardos-Benachour [3] and Beale-Kato-Majda [5]), this implies that the solution of the Euler equation exists, at least, up to time $\theta_{3}$.

The final part of our analysis concerns an alternative approach to estimate $\theta_{3}$, and the possibility that $u(t)$ blows up at times larger than $\theta_{3}$. In connection with this problem we use the idea (employed in $[9,13,17,22]$ for different initial data) to construct the Padé approximants for the (squared) Sobolev norms and analyze their singularities. In particular, we construct the diagonal Padé approximants $[p / p](t)$ for $\|u(t)\|_{3}^{2}$, up to $p=26$, and some near-diagonal appproximants $[p / q](t)$ (with $p+q=50$ or 52 ). For most of them the complex 
singularities of minimum modulus have modulus $\simeq 0.5$; this fact yields new evidence for the previous estimate on $\theta_{3}$. Moreover, most of these Padé approximants have real or "almost real" singularities, distributed rather erratically; analyzing these distributions in terms of mean values and mean quadratic errors, we obtain a somehow weak indication that:

(c) $u(t)$ might blow up for $t \rightarrow T^{-}$(and $t \rightarrow(-T)^{+}$), for some $T$ such that $0.54<T<0.85$ (see Eq. (6.7)).

The blow-up problem can be studied as well in terms of D-log Padé approximants; these do not give a clear indication supporting conjecture (c), as briefly explained at the end of the paper. In general, much caution is recommended about the Euler equation and blow-up predictions via Padé analysis: for example, in the case of the Taylor-Green vortex the Padé approximants exhibit real singularities [13,17], but the numerical solution of the Euler equation by spectral methods raises doubts on the actual existence of a blow-up $[8,10,14]$.

Connections with other works. Concluding this Introduction, to put the subject of this paper into a wider perspective we wish to mention that there are general methods of functional analysis to obtain quantitive lower bounds on the time of existence $T$ of the solution of the Euler (or Navier-Stokes) Cauchy problem, from the a posteriori analysis of an approximate solution; such lower bounds are certain (i.e., non conjectural).

Derivations of such a posteriori lower bounds have been given in $[12,19,21]$. The last of these works gives an algorithm to obtain these lower bounds analyzing any approximate solution of the Euler (or Navier-Stokes) Cauchy problem via a suitable differential inequality, called therein the "control inequality".

Again in [21], a preliminary analysis of the Euler (and Navier-Stokes) equations with the Behr-Nečas-Wu initial datum has been performed, using for the solution a Galerkin approximation with very few Fourier modes. This approximant, combined with the control inequality, gives for the Euler equation with this datum a (poor, but certain) lower bound $T>0.066$ for the time of existence (in $H^{3}$; the same approach, applied to the Navier-Stokes equations, grants $T=+\infty$ when the viscosity coefficient is above an explicit threshold). We plan to continue in future works the analysis of the Behr-Nečas-Wu intial datum, combining the control equation of [21] with approximation methods based on extensive automatic computations such as the ones presented in this paper.

\section{The Cauchy problem for the Euler EQuation on A torus}

Preliminaries. If $a=\left(a_{s}\right), b=\left(b_{s}\right)$ are elements of $\mathbf{R}^{3}$ or $\mathbf{C}^{3}$, we define $a \bullet b:=\sum_{s=1}^{3} a_{s} b_{s}$. We indicate with ${ }^{-}$ the complex conjugate (and we let it act componentwise on elements of $\mathbf{C}^{3}$ ); we put $|a|:=\sqrt{\bar{a} \bullet a}=\sqrt{\sum_{s=1}^{3}\left|a_{s}\right|^{2}}$.

The Cauchy problem for the incompressible Euler equation is

$$
\frac{\partial u}{\partial t}=-u \bullet \nabla u-\nabla p, \quad u(x, 0)=u_{0}(x),
$$

where: $u=u(x, t)$ is the divergence-free velocity field; the space variables $x=\left(x_{s}\right)_{s=1,2,3}$ belong to the torus $\mathbf{T}^{3}:=(\mathbf{R} / 2 \pi \mathbf{Z})^{3} ;(u \bullet \nabla u)_{r}:=\sum_{s=1}^{3} u_{s} \partial_{s} u_{r}(r=1,2,3) ; p=p(x, t)$ is the pressure; $u_{0}=u_{0}(x)$ is the initial datum. As well known, the pressure can be eliminated from (2.1) using the Leray projection $\mathfrak{L}$ onto the space of divergence-free vector fields; this allows to rewrite the evolution equation in $(2.1)$ as $\partial u / \partial t=-\mathfrak{L}(u \bullet \nabla u)$. In this way, we obtain for the Cauchy problem the final form

$$
\frac{\partial u}{\partial t}=\mathcal{P}(u, u), \quad u(., 0)=u_{0},
$$

where we have written $\mathcal{P}$ for the bilinear map sending two (sufficiently regular) vector fields $v, w: \mathbf{T}^{3} \rightarrow \mathbf{R}^{3}$ into the vector field

$$
\mathcal{P}(v, w):=-\mathfrak{L}(v \bullet \nabla w) .
$$


In this framework, it is convenient to associate to a vector field $v: \mathbf{T}^{3} \rightarrow \mathbf{R}^{3}$ the Fourier components $v_{k}:=$ $(2 \pi)^{-3} \int_{\mathbf{T}^{3}} \mathrm{~d} x \mathrm{e}^{-i k \bullet x} v(x) \in \mathbf{C}^{3}$, so that

$$
v(x)=\sum_{k \in \mathbf{Z}^{3}} v_{k} \mathrm{e}^{i k \bullet x} .
$$

Due to the reality of $v$, we have $v_{-k}=\overline{v_{k}}$, and $v$ is divergence-free iff $k \bullet v_{k}=0$ for all $k$. With $\mathcal{P}$ as above and $v, w$ two vector fields, the Fourier components of $\mathcal{P}(v, w)$ are

$$
\mathcal{P}(v, w)_{k}=-i \sum_{h \in \mathbf{Z}^{3}} v_{h} \bullet(k-h) \mathfrak{L}_{k} w_{k-h}
$$

where $\mathfrak{L}_{k}: \mathbf{C}^{3} \rightarrow \mathbf{C}^{3}$ is the projection on the orthogonal complement of $k\left(\mathfrak{L}_{k} c:=c-(k \bullet c) k /|k|^{2}\right.$ if $k \neq 0$; $\left.\mathfrak{L}_{0} c:=c\right)$.

In the above, we have introduced the setting for the Euler equation in an informal way; to go on, it is necessary to specify the functional spaces to which the velocity fields (at any time) are supposed to belong.

The expression "a vector field $\mathbf{T}^{3} \rightarrow \mathbf{R}^{3}$ " can be understood, with very wide generality, as "an $\mathbf{R}^{3}$-valued distribution on $\mathbf{T}^{3}$ " (see, e.g., $[20]$ ); we write $D^{\prime}\left(\mathbf{T}^{3}, \mathbf{R}^{3}\right) \equiv D^{\prime}$ for the space of such distributions. Any $v \in$ $D^{\prime}\left(\mathbf{T}^{3}, \mathbf{R}^{3}\right)$ can be differentiated in the distributional sense and has a (weakly convergent) Fourier expansion with coefficients $v_{k} \in \mathbf{C}^{3}$, such that $\overline{v_{k}}=v_{-k}$.

To construct the full setting for the Euler equation, one must confine the attention to much smaller functional spaces of vector fields. For our purposes, two cases are important:

(i) The Sobolev space $H^{n}$ of zero mean, divergence-free vector fields of any order $n \in[0,+\infty)$. This is defined in terms of the space $L^{2}\left(\mathbf{T}^{3}, \mathbf{R}^{3}\right) \equiv L^{2}$ of square integrable vector fields $v: \mathbf{T}^{3} \rightarrow \mathbf{R}^{3}$, equipped with the inner product $\langle v \mid w\rangle_{L^{2}}:=(2 \pi)^{-3} \int_{\mathbf{T}^{3}} v(x) \bullet w(x) \mathrm{d} x$ and with the induced norm $\|v\|_{L^{2}}=(2 \pi)^{-3 / 2} \sqrt{\int_{\mathbf{T}^{3}}|v(x)|^{2} \mathrm{~d} x}$ (note the term $(2 \pi)^{-3}$ in the inner product, used systematically in the sequel). By definition,

$$
\begin{gathered}
H^{n}\left(\mathbf{T}^{3}, \mathbf{R}^{3}\right) \equiv H^{n}:=\left\{v \in D^{\prime} \mid \sqrt{-\Delta}^{n} v \in L^{2}, \int_{\mathbf{T}^{3}} v \mathrm{~d} x=0, \operatorname{div} v=0\right\} \\
=\left\{\left.v \in D^{\prime}\left|\sum_{k \in \mathbf{Z}^{3}}\right| k\right|^{2 n}\left|v_{k}\right|^{2}<+\infty, v_{0}=0, k \bullet v_{k}=0\right\} .
\end{gathered}
$$

(In the above $\sqrt{-\Delta}^{n}$ indicates the power of order $n / 2$ of minus the Laplacian; by definition $\left(\sqrt{-\Delta}^{n} v\right)_{k}=$ $|k|^{n} v_{k}$ for each $v \in D^{\prime}$. Note that $H^{n} \subset L^{2}$ for all $\left.n \geqslant 0\right)$. $H^{n}$ is a Hilbert space with the inner product

$$
\langle v \mid w\rangle_{n}:=\left\langle\sqrt{-\Delta}^{n} v \mid \sqrt{-\Delta}^{n} w\right\rangle_{L^{2}}=\sum_{k \in \mathbf{Z}^{3}}|k|^{2 n} \overline{v_{k}} \bullet w_{k},
$$

inducing the norm

$$
\|v\|_{n}=\left\|\sqrt{-\Delta}^{n} v\right\|_{L^{2}}=\sqrt{\sum_{k \in \mathbf{Z}^{3}}|k|^{2 n}\left|v_{k}\right|^{2}} .
$$

It is known that $\mathcal{P}$ sends continuously $H^{n} \times H^{n+1}$ into $H^{n}$, for all $n \in(3 / 2,+\infty)$.

(ii) The space of $C^{\omega}$ (i.e., analytic) zero mean, divergence-free vector fields on $\mathbf{T}^{3}$; this is

$$
\begin{aligned}
& \mathcal{A}\left(\mathbf{T}^{3}, \mathbf{R}^{3}\right) \equiv \mathcal{A}:=\left\{v \in C^{\omega}\left(\mathbf{T}^{3}, \mathbf{R}^{3}\right) \mid \int_{\mathbf{T}^{3}} v \mathrm{~d} x=0, \operatorname{div} v=0\right\} \\
& =\left\{\left.v \in D^{\prime}\left|\liminf _{k \in \mathbf{Z}^{3}, k \rightarrow \infty}\right| v_{k}\right|^{-\frac{1}{\left|k_{1}\right|+\left|k_{2}\right|+\left|k_{3}\right|}}>1, v_{0}=0, k \bullet v_{k}=0\right\}
\end{aligned}
$$


(defining $0^{-\frac{1}{\left|k_{1}\right|+\left|k_{2}\right|+\left|k_{3}\right|}}:=+\infty$. The Fourier representation in (2.9) mimics the description of analytic functions on the torus in [18], which is also a useful reference for what follows). One has

$$
\begin{gathered}
\mathcal{A}=\cup_{\rho \in(1,+\infty)} \mathcal{A}_{\rho}, \\
\mathcal{A}_{\rho}:=\left\{\left.v \in D^{\prime}\left|\liminf _{k \in \mathbf{Z}^{3}, k \rightarrow \infty}\right| v_{k}\right|^{-\frac{1}{\left|k_{1}\right|+\left|k_{2}\right|+\left|k_{3}\right|}}>\rho, v_{0}=0, \quad k \bullet v_{k}=0\right\} ;
\end{gathered}
$$

each $\mathcal{A}_{\rho}$ is a vector subspace of $\mathcal{A}$. Let us introduce the annulus $K_{\rho}:=\{z \in \mathbf{C}|1 / \rho \leqslant| z \mid \leqslant \rho\}$ and its power $K_{\rho}^{3}:=\left\{z=\left(z_{1}, z_{2}, z_{3}\right) \in \mathbf{C}^{3} \mid z_{1}, z_{2}, z_{3} \in K_{\rho}\right\}$. For $v \in \mathcal{A}_{\rho}$, the series $\sum_{k \in \mathbf{Z}^{3}} v_{k} z^{k}$ converges in $\mathbf{C}^{3}$ for each $z \in K_{\rho}^{3}$ (defining $z^{k}:=z_{1}^{k_{1}} z_{2}^{k_{2}} z_{3}^{k_{3}}$ ); the function $z \mapsto \sum_{k \in \mathbf{Z}^{3}} v_{k} z^{k}$ is holomorphic on the inner part of $K_{\rho}^{3}$ and continuous on $K_{\rho}^{3}$, so we can define

$$
\left\|v\left|\|_{\rho}:=\sup _{z \in K_{\rho}^{3}}\right| \sum_{k \in \mathbf{Z}^{3}} v_{k} z^{k} \mid .\right.
$$

\|I \|\|$_{\rho}$ is a norm on $\mathcal{A}_{\rho}$ and makes it a Banach space. One equips $\mathcal{A}$ with the inductive limit topology of the collection of Banach spaces $\left\{\left(\mathcal{A}_{\rho},\|\mid\| \|_{\rho}\right) \mid \rho \in(1,+\infty)\right\}$ : this is the finest locally convex topology on $\mathcal{A}$ making continuous each embedding $\mathcal{A}_{\rho} \hookrightarrow \mathcal{A}$. (Besides [18], see [25] for the general theory of inductive limits). $\mathcal{A}$ is continuously embedded into each Sobolev space $H^{n}$; the map $\mathcal{P}$ is continuous from $\mathcal{A} \times \mathcal{A}$ to $\mathcal{A}$.

Basic results on local existence and uniqueness. We start from the Sobolev framework, choosing

$$
n \in(5 / 2,+\infty) .
$$

In the sequel, an $H^{n}$-solution of the Euler equation, or of the Euler Cauchy problem, means a map

$$
u \in C\left((-\mathcal{T}, T), H^{n}\right) \cap C^{1}\left((-\mathcal{T}, T), H^{n-1}\right)
$$

$(\mathcal{T}, T \in(0,+\infty])$ fulfilling the Euler equation, or its Cauchy problem with a suitable initial condition $u(0)=u_{0}$. The following statement is well known:

Proposition 2.1. For $n$ as above and any initial datum $u_{0} \in H^{n}$, the following holds.

(i) The Cauchy problem (2.2) has a unique maximal (i.e., not extendable) $H^{n}$-solution u of domain $(-\mathcal{T}, T)$, for suitable $T=T\left(u_{0}\right), \mathcal{T}=\mathcal{T}\left(u_{0}\right) \in(0,+\infty]$. Any $H^{n}$-solution of $(2.2)$ is a restriction of the maximal one.

(ii) If $T<+\infty$, one has

$$
\int_{0}^{T} \mathrm{~d} t\|u(t)\|_{n}=+\infty
$$

a fact implying

$$
\limsup _{t \rightarrow T^{-}}\|u(t)\|_{n}=+\infty .
$$

Similar results hold if $\mathcal{T}<+\infty$, considering the integral from $-\mathcal{T}$ to 0 (and the limit for $t \rightarrow(-\mathcal{T})^{+}$).

Proof.

(i) This follows, e.g., from Kato's theory of quasilinear evolution equations [15].

(ii) The thesis can be inferred from a known result of Beale-Kato-Majda [5]. The cited paper shows that $T<$ $+\infty$ implies $\int_{0}^{T} \mathrm{~d} t\|\operatorname{rot} u(t)\|_{L^{\infty}}=+\infty$; however, $\|\operatorname{rot} u(t)\|_{L^{\infty}} \leqslant$ const. $\|u(t)\|_{n}$ by the Sobolev imbedding inequalities, whence equation (2.14). The behavior of $u$ at $-\mathcal{T}$ is analyzed similarly.

If $T<+\infty$, the solution $u$ is said to blow up at time $T$. Similarly, if $\mathcal{T}<+\infty$ we say that $u$ blows up at $-\mathcal{T}$. Many statements presented in the sequel on the possibility of blow-up at $T$ have obvious reformulations regarding $-\mathcal{T}$. 
Remark 2.2. The blow-up criterion (2.14) yields the following statement, in case of blow-up with a power law:

$$
\text { if }\|u(t)\|_{n} \sim \frac{U}{(T-t)^{\alpha}} \text { for } t \rightarrow T^{-}(\text {with } U, \alpha>0), \quad \text { then } \alpha \geqslant 1 .
$$

In the case of the Euler equation on $\mathbf{R}^{3}$, it was recently shown in [11], Theorem 1.3 that the blow-up at $T$ implies the following (for any $n>5 / 2$ ):

$$
\|u(t)\|_{n} \geqslant \frac{U}{(T-t)^{2 n / 5}} \quad \text { for } t \text { close to } T, \quad U=U_{n}\left(\left\|u_{0}\right\|_{L^{2}}\right) .
$$

This estimate might hold as well for the framework of the present paper, i.e., for the Euler equation on the torus $\mathbf{T}^{3}$ (however, the extendability of (2.17) to $\mathbf{T}^{3}$ is immaterial for the purposes of this paper).

Let us pass to the $C^{\omega}$ (= analytic) framework; what follows assumes some general notions from the theory of analytic functions from $\mathbf{R}$ to locally convex spaces, for which we refer to [7], Section 3. Let $\mathcal{A}$ be the space (2.9); in the sequel, an $\mathcal{A}$-solution of the Euler equation, or of the Euler Cauchy problem, means a map

$$
u \in C^{\omega}((-\mathcal{T}, T), \mathcal{A})
$$

$(\mathcal{T}, T \in(0,+\infty])$ fulfilling the Euler equation, or its Cauchy problem with a suitable initial condition $u(0)=u_{0}$. Here is a known statement (with some indications on the proof, given only for completeness):

Proposition 2.3. Consider an initial datum $u_{0} \in \mathcal{A}$; then the following holds.

(i) For any $n \in(5 / 2,+\infty)$, a function $u$ is an $\mathcal{A}$-solution of the Cauchy problem (2.2) if and only if $u$ is an $H^{n}$-solution.

(ii) Problem (2.2) has a unique maximal (i.e., non extendable) $\mathcal{A}$-solution $u$ of domain $(-\mathcal{T}, T)$, for suitable $T=T\left(u_{0}\right), \mathcal{T}=\mathcal{T}\left(u_{0}\right) \in(0,+\infty]$ (and any other $\mathcal{A}$-solution is a restriction of $u$ ). For any $n \in(5 / 2,+\infty)$, $u$ coincides with the maximal $H^{n}$-solution (and thus, if $T<+\infty$, it fulfills equations (2.14) and (2.15); a similar result holds if $\mathcal{T}<+\infty)$.

Proof.

(i) Assume $u$ is an $\mathcal{A}$-solution of the Cauchy problem (2.2); then, by the continuous imbedding of $\mathcal{A}$ into any Sobolev space, $u$ is as well an $H^{n}$-solution.

Conversely, assume $u$ to be an $H^{n}$-solution of (2.2), of domain $(-\mathcal{T}, T)$. Then $\partial u / \partial t \in C\left((-\mathcal{T}, T), H^{n-1}\right) \subset$ $C((-\mathcal{T}, T), \mathcal{C})$ and $u \in C\left((-\mathcal{T}, T), H^{n}\right) \subset C\left((-\mathcal{T}, T), \mathcal{C}^{1}\right)$ where $\mathcal{C}:=C\left(\mathbf{T}^{3}, \mathbf{R}^{3}\right), \mathcal{C}^{1}:=C^{1}\left(\mathbf{T}^{3}, \mathbf{R}^{3}\right)$ and we have used the imbeddings of $H^{n-1}$ and $H^{n}$ into $\mathcal{C}$ and $\mathcal{C}^{1}$, respectively. The previous statements ensure that the function $(x, t) \mapsto u(x, t)$ is $C^{1}$ from $(-\mathcal{T}, T) \times \mathbf{T}^{3}$ to $\mathbf{R}^{3}$. Since this function fulfills the Euler equation with an analytic initial datum, due to Theorem II.2, page 667 of Bardos-Benachour [3] we have $u(t) \in \mathcal{A}$ for each $t$; moreover, the cited work ensures that $u \in C^{1}((-\mathcal{T}, T), \mathcal{A})$ and this fact, combined with Theorem III.2, page 264 of Baouendi-Goulaouic [2], implies $u \in C^{\omega}((-\mathcal{T}, T), \mathcal{A})$. In conclusion $u$ is as well an $\mathcal{A}$-solution of the Euler Cauchy problem.

(ii) All statements in this item can be proved using the equivalence between the notions of $\mathcal{A}$ - and $H^{n}$ - solution (known from item (i)), together with Proposition 2.1 on the $H^{n}$-solutions $(n>5 / 2)\left({ }^{5}\right)$.

\footnotetext{
${ }^{5}$ Of course, a more elegant proof of existence (and uniqueness) in the framework of $\mathcal{A}$-solutions is a direct one, not relying on Proposition 2.1. Proofs of this type are given in Bardos-Benachour [3] (see Thm. II.1, p. 665) and Baouendi-Goulaouic [2] (see Thm. III.2, p. 264). For completeness, let us mention that a statement very close to the contents of Proposition 2.3 (namely, the equivalence between the loss of analiticity and the Beale-Kato-Majda blow-up criterion for the integral of $\left.\|\operatorname{rot} u(t)\|_{L^{\infty}}\right)$ appears without proof in Bardos-Titi [4], Remark 2.1, pages 414.
} 
Assume again that $u_{0} \in \mathcal{A}$ and $u$ is the maximal $\mathcal{A}$-solution of the Euler Cauchy problem; let us choose any $n \in[0,+\infty)$. For future use, it is convenient to record the following facts:

(a) $u \in C^{\omega}\left((-\mathcal{T}, T), H^{n}\right)$ for the already mentioned continuous embedding of $\mathcal{A}$ into any Sobolev space;

(b) the function

$$
(-\mathcal{T}, T) \rightarrow \mathbf{R}, \quad t \mapsto\|u(t)\|_{n}^{2}
$$

is in $C^{\omega}((-\mathcal{T}, T), \mathbf{R})$, being the composition of the analytic function $u:(-\mathcal{T}, T) \rightarrow H^{n}$ with the continuous quadratic function \|\|$_{n}^{2}: H^{n} \rightarrow \mathbf{R}$.

Symmetries of the Euler equation. Let us consider the octahedral group $O_{h}$, formed by the orthogonal $3 \times 3$ matrices with integer entries:

$$
O_{h}:=\left\{S \in \operatorname{Mat}(3 \times 3, \mathbf{Z}) \mid S^{\mathrm{T}} S=\mathbf{1}\right\} .
$$

In fact, the entries of any such matrix have $-1,0$ and 1 as the only possible values; furthermore, a $3 \times 3$ matrix $S$ belongs to $O_{h}$ if and only if

$$
S=\operatorname{diag}\left(\epsilon_{1}, \epsilon_{2}, \epsilon_{3}\right) Q(\sigma)
$$

$$
\epsilon_{s} \in\{ \pm 1\}(s=1,2,3) ; Q(\sigma) \text { the matrix of the permutation } \sigma:\{1,2,3\} \rightarrow\{1,2,3\} ;
$$

more precisely, $Q(\sigma)$ is the matrix such that $(Q(\sigma) c)_{s}=c_{\sigma(s)}$ for all $c \in \mathbf{C}^{3}, s \in\{1,2,3\}$. There are $2^{3}=8$ possible choices for the signs $\epsilon_{i}$ and $3 !=6$ choices for $\sigma$, so $O_{h}$ has $8 \times 6=48$ elements. Clearly, each $S \in O_{h}$ sends $\mathbf{Z}^{3}$ into itself.

To go on, let us denote with $O_{h} \ltimes \mathbf{T}^{3}$ the Cartesian product $O_{h} \times \mathbf{T}^{3}$, viewed as a group with the composition law defined by $\left({ }^{6}\right)$

$$
(S, a)(U, b):=(S U, a+S b) \quad\left(S, U \in O_{h} ; a, b \in \mathbf{T}^{3}\right) .
$$

Of course, the unit of this group is $(\mathbf{1}, 0)$ (with $\mathbf{1}$ denoting again the identity $3 \times 3$ matrix); the inverse of a pair $(S, a)$ is $(S, a)^{-1}=\left(S^{\mathrm{T}},-S^{\mathrm{T}} a\right)$. To any element $(S, a)$ of $O_{h} \ltimes \mathbf{T}^{3}$ is associated a "rototranslation"

$$
\mathcal{E}(S, a): \mathbf{T}^{3} \rightarrow \mathbf{T}^{3}, \quad x \mapsto \mathcal{E}(S, a)(x):=S x+a,
$$

and one checks that the mapping $(S, a) \mapsto \mathcal{E}(S, a)$ is a group homomorphism between $O_{h} \ltimes \mathbf{T}^{3}$ and the group of diffeomorphisms of $\mathbf{T}^{3}$ into itself (with the usual composition).

Now, we take a vector field $v$ in $H^{n}$ (or in $\mathcal{A}$ ) and an element $(S, a)$ of the group $O_{h} \ltimes \mathbf{T}^{3}$. We can construct the push-forward $\mathcal{E}_{*}(S, a) v$ of $v$ along the mapping $\mathcal{E}(S, a)$; this is the vector field in $H^{n}$ (or in $\mathcal{A}$ ), given by

$$
\mathcal{E}_{*}(S, a) v: \mathbf{T}^{3} \rightarrow \mathbf{R}^{3}, \quad x \mapsto\left(\mathcal{E}_{*}(S, a) v\right)(x)=S v\left(S^{\mathrm{T}}(x-a)\right) .
$$

One easily checks that Eq. (2.24) actually defines a vector field in $H^{n}$ (or in $\mathcal{A}$ ), with Fourier components

$$
\left(\mathcal{E}_{*}(S, a) v\right)_{k}=\mathrm{e}^{-i a \bullet k} S v_{S^{\mathrm{T}}{ }_{k}} \quad\left(k \in \mathbf{Z}^{3}\right) .
$$

Let us write $\mathcal{E}_{*}(S, a)$ for the map $v \in H^{n} \mapsto \mathcal{E}_{*}(S, a) v$; this is a linear map of $H^{n}$ into itself, preserving the inner product $\langle\mid\rangle_{n}$, so it is in the group $O\left(H^{n}\right)$ of orthogonal operators of the Hilbert space $H^{n}$ into itself. The mapping

$$
\mathcal{E}_{*}: O_{h} \ltimes \mathbf{T}^{3} \rightarrow O\left(H^{n}\right), \quad(S, a) \mapsto \mathcal{E}_{*}(S, a)
$$

is a injective group homomorphism, i.e., a faithful orthogonal representation of the group $O_{h} \ltimes \mathbf{T}^{3}$ on the real Hilbert space $H^{n}$. Alternatively, let us write $\mathcal{E}_{*}(S, a)$ for the map $v \in \mathcal{A} \mapsto \mathcal{E}_{*}(S, a) v$; this is in the $\operatorname{space} \operatorname{Iso}(\mathcal{A})$ of linear and topological isomorphisms of $\mathcal{A}$ into itself. The map

$$
\mathcal{E}_{*}: O_{h} \ltimes \mathbf{T}^{3} \rightarrow \operatorname{Iso}(\mathcal{A}), \quad(S, a) \mapsto \mathcal{E}_{*}(S, a)
$$

\footnotetext{
${ }^{6}$ This is the semidirect product of the groups $O_{h}$ and $\mathbf{T}^{3}$ with respect to the natural homomorphism $O_{h} \rightarrow A u t\left(\mathbf{T}^{3}\right)$ sending $S \in O_{h}$ into the map $b \mapsto S b$, an automorphism of $\mathbf{T}^{3}$.
} 
is an injective group homomorphism, i.e., a faithful linear representation of the group $O_{h} \ltimes \mathbf{T}^{3}$ on the topological vector space $\mathcal{A}$.

Let us relate the previous constructions to the bilinear map $\mathcal{P}$ of the Euler equation. From the Fourier representations (2.5)-(2.25), one easily infers

$$
\mathcal{P}\left(\mathcal{E}_{*}(S, a) v, \mathcal{E}_{*}(S, a) w\right)=\mathcal{E}_{*}(S, a) \mathcal{P}(v, w)
$$

for all $v \in H^{n}, w \in H^{n+1}$ with $n>3 / 2$ (and, in particular, for all $v, w \in \mathcal{A}$ ). Let us outline the implications of (2.28) about the solutions of the Euler equation. In the rest of the paragraph, the term "solution" either means an $H^{n}$-solution $(n>5 / 2)$ or an $\mathcal{A}$-solution, and the initial datum $u_{0}$ is chosen consistently in $H^{n}$ or in $\mathcal{A}$. From (2.28) one infers the following, for each $(S, a) \in O_{h} \ltimes \mathbf{T}^{3}$ :

(i) If $u: t \in(-\mathcal{T}, T) \mapsto u(t)$ is a solution of the Euler equation, we have two more solutions

$$
\begin{gathered}
\mathcal{E}_{*}(S, a) u: t \in(-\mathcal{T}, T) \mapsto \mathcal{E}_{*}(S, a) u(t), \\
-\mathcal{E}_{*}(S, a) u(-\cdot): t \in(-T, \mathcal{T}) \mapsto-\mathcal{E}_{*}(S, a) u(-t) .
\end{gathered}
$$

(ii) If $u: t \in(-\mathcal{T}, T) \mapsto u(t)$ is the maximal solution of the Euler Cauchy problem with datum $u_{0}$, then $\mathcal{E}_{*}(S, a) u$ is the maximal solution with datum $\mathcal{E}_{*}(S, a) u_{0}$ and $-\mathcal{E}_{*}(S, a) u(-\cdot)$ is the maximal solution with datum $-\mathcal{E}_{*}(S, a) u_{0}$.

(iii) Let us denote again with $u: t \in(-\mathcal{T}, T) \mapsto u(t)$ the maximal solution of the Cauchy problem with datum $u_{0}$. Then,

$$
\begin{gathered}
\mathcal{E}_{*}(S, a) u_{0}=u_{0} \Rightarrow \mathcal{E}_{*}(S, a) u(t)=u(t) \quad \text { for } t \in(-\mathcal{T}, T) . \\
-\mathcal{E}_{*}(S, a) u_{0}=u_{0} \Rightarrow \mathcal{T}=T,-\mathcal{E}_{*}(S, a) u(-t)=u(t) \quad \text { for } t \in(-T, T) .
\end{gathered}
$$

The verification of statements (i)(ii) is straightforward. After this, the implication (2.31) in (iii) follows noting that $\mathcal{E}_{*}(S, a) u$ and $u$ are maximal solutions of the Cauchy problem with the same datum $\mathcal{E}_{*}(S, a) u_{0}=u_{0}$. Similarly, the implication (2.32) follows noting that $-\mathcal{E}_{*}(S, a) u(-\cdot)$ and $u$ are maximal solutions of the Cauchy problem with the same datum $-\mathcal{E}_{*}(S, a) u_{0}=u_{0}$.

Considering the maximal solution $u$ for a datum $u_{0}$ in $H^{n}(n>5 / 2)$, and recalling that any transformation $\mathcal{E}_{*}(S, a)$ preserves the $H^{n}$ norm, we also obtain from (2.32) the following:

$$
-\mathcal{E}_{*}(S, a) u_{0}=u_{0} \Rightarrow \mathcal{T}=T,\|u(-t)\|_{n}=\|u(t)\|_{n} \quad \text { for } t \in(-T, T) .
$$

The results in (iii) suggest to consider, for a given datum $u_{0}$ in $H^{n}$ or $\mathcal{A}$, the symmetry subgroup

$$
\mathcal{H}\left(u_{0}\right):=\left\{(S, a) \in O_{h} \ltimes \mathbf{T}^{3} \mid \mathcal{E}_{*}(S, a) u_{0}=u_{0}\right\}
$$

and the pseudo-symmetry space

$$
\mathcal{H}^{-}\left(u_{0}\right):=\left\{(S, a) \in O_{h} \ltimes \mathbf{T}^{3} \mid-\mathcal{E}_{*}(S, a) u_{0}=u_{0}\right\}
$$

(the first one, being a subgroup of $O_{h} \ltimes \mathbf{T}^{3}$, contains at least the identity element $(\mathbf{1}, 0)$; the second one might be the empty set. The term "isotropy group", often employed in place of "symmetry group", will not be used in this paper). 
Let us consider the maximal solution $u$ of the Cauchy problem with a datum $u_{0}$ (contained in $H^{n}$ for some $n>5 / 2)$; from equations (2.31)-(2.33), we readily obtain the following:

$$
\begin{gathered}
\mathcal{E}_{*}(S, a) u(t)=u(t) \quad \text { for all }(S, a) \in \mathcal{H}\left(u_{0}\right), t \in(-\mathcal{T}, T) \\
\mathcal{H}^{-}\left(u_{0}\right) \neq \emptyset \Rightarrow \quad \mathcal{T}=T,-\mathcal{E}_{*}(S, a) u(-t)=u(t),\|u(-t)\|_{n}=\|u(t)\|_{n} \\
\\
\quad \text { for }(S, a) \in \mathcal{H}^{-}\left(u_{0}\right), t \in(-T, T) .
\end{gathered}
$$

For future use, let us introduce the reduced symmetry subgroup and the reduced pseudo-symmetry space of the datum $u_{0}$, which are

$$
\begin{gathered}
\mathcal{H}_{R}\left(u_{0}\right):=\left\{S \in O_{h} \mid \mathcal{E}_{*}(S, a) u_{0}=u_{0} \text { for some } a \in \mathbf{T}^{3}\right\}, \\
\mathcal{H}_{R}^{-}\left(u_{0}\right):=\left\{S \in O_{h} \mid-\mathcal{E}_{*}(S, a) u_{0}=u_{0} \text { for some } a \in \mathbf{T}^{3}\right\} .
\end{gathered}
$$

Let us observe that the set theoretical unions $\mathcal{H}\left(u_{0}\right) \cup \mathcal{H}^{-}\left(u_{0}\right)$ and $\mathcal{H}_{R}\left(u_{0}\right) \cup \mathcal{H}_{R}^{-}\left(u_{0}\right)$ are subgroups of $O_{h} \ltimes \mathbf{T}^{3}$ and $O_{h}$, respectively.

As a final remark, useful for the sequel, let us consider the pair $(-\mathbf{1}, 0) \in O_{h} \ltimes \mathbf{T}^{3}$, noting that $\mathcal{E}(-\mathbf{1}, 0)$ is the space reflection: $\mathcal{E}(-\mathbf{1}, 0)(x)=-x$ for all $x \in \mathbf{T}^{3}$. One easily checks that

$$
(-1,0) \in \mathcal{H}^{-}\left(u_{0}\right) \Leftrightarrow \mathcal{H}^{-}\left(u_{0}\right)=\mathcal{H}\left(u_{0}\right)(-1,0)=\left\{(-S, a) \mid(S, a) \in \mathcal{H}\left(u_{0}\right)\right\}
$$

(where $\mathcal{H}\left(u_{0}\right)(-\mathbf{1}, 0)$ stands for the set $\left\{(S, a)(-\mathbf{1}, 0) \mid(S, a) \in \mathcal{H}\left(u_{0}\right)\right\}$; the last equality rests on the identity $(S, a)(-\mathbf{1}, 0)=(-S, a))$.

\section{Power series in time for the Euler Cauchy problem}

Throughout this section, we consider the Euler Cauchy problem with initial datum $u_{0} \in \mathcal{A}$.

Setting up a power series for the solution. Let us try to build the solution of the Euler Cauchy problem as a power series

$$
t \mapsto \sum_{j=0}^{\infty} u_{j} t^{j}
$$

with coefficients $u_{j} \in \mathcal{A}$, whose convergence has to be discussed later. The zero order term in this expansion is the initial datum $u_{0}$; to determine the other coefficients $u_{j} \in \mathcal{A}$, it suffices to substitute the expansion (3.1) into the Euler equation (2.2), and to require equality of the coefficients of the same powers of $t$ in both sides: in this way, one easily obtains the recurrence relation

$$
u_{j}=\frac{1}{j} \sum_{\ell=0}^{j-1} \mathcal{P}\left(u_{\ell}, u_{j-\ell-1}\right) \quad(j=1,2,3, \ldots) .
$$

When applying this recurrence relation for the $u_{j}$ 's it can be useful to represent the bilinear map $\mathcal{P}$ in terms of Fourier coefficients, as in equation (2.5). This is especially useful if the initial datum $u_{0}$ is a Fourier polynomial, i.e., if $u_{0 k} \neq 0$ only for finitely many modes $k$. In this case, all the iterates $u_{j}(j=1,2,3, \ldots)$ are as well Fourier polynomials, and the implementation of (3.2) via the Fourier representation (2.5) always involves sums over finitely many modes.

In the next section, a large part of our attention will be devoted (for a specific datum $u_{0}$ ) to the partial sums

$$
u^{(N)}(t):=\sum_{j=0}^{N} u_{j} t^{j}
$$


$(N=0,1,2, \ldots)$ and to the (squared) Sobolev norms

$$
\left\|u^{(N)}(t)\right\|_{n}^{2}=\sum_{k \in \mathbf{Z}^{3}}|k|^{2 n}\left|u_{k}^{(N)}(t)\right|^{2} .
$$

Symmetry considerations. Let us consider the symmetry subgroup $\mathcal{H}\left(u_{0}\right)$ or the pseudo-symmetry space $\mathcal{H}^{-}\left(u_{0}\right)$, see equations (2.34)-(2.35). Using the recursive definition (3.2) of $u_{j}$ with the invariance property $(2.28)$ of $\mathcal{P}$, one easily checks the following, for any $j \in\{0,1,2, \ldots\}$ :

$$
\begin{gathered}
\mathcal{E}_{*}(S, a) u_{j}=u_{j} \quad \text { for all }(S, a) \in \mathcal{H}\left(u_{0}\right) ; \\
-\mathcal{E}_{*}(S, a) u_{j}=(-1)^{j} u_{j} \quad \text { for all }(S, a) \in \mathcal{H}^{-}\left(u_{0}\right) .
\end{gathered}
$$

Of course, the last two equations imply the following, for all $N \in\{0,1,2, \ldots\}, t \in \mathbf{R}$ and $n \in[0,+\infty)$ :

$$
\begin{gathered}
\mathcal{E}_{*}(S, a) u_{N}(t)=u_{N}(t) \quad \text { for }(S, a) \in \mathcal{H}\left(u_{0}\right) ; \\
-\mathcal{E}_{*}(S, a) u_{N}(t)=u_{N}(-t) \quad \text { for }(S, a) \in \mathcal{H}^{-}\left(u_{0}\right) ; \\
\left\|u_{N}(t)\right\|_{n}=\left\|u_{N}(-t)\right\|_{n} \quad \text { if } \mathcal{H}^{-}\left(u_{0}\right) \neq \emptyset
\end{gathered}
$$

(Eq. (3.9) is a consequence of Eq. (3.8) and of the invariance of \|\|$_{n}$ under the transformation $\mathcal{E}_{*}(S, a)$ ).

Due to the Fourier representation $(2.25)$ for $\mathcal{E}_{*}(S, a)$, the equality $(3.5)$ reads $\mathrm{e}^{-i a \bullet k} S u_{j, S^{\mathrm{T}} k}=u_{j, k}$ or, equivalently,

$$
u_{j, S k}=\mathrm{e}^{-i a \bullet S k} S u_{j, k} \quad \text { for } k \in \mathbf{Z}^{3},(S, a) \in \mathcal{H}\left(u_{0}\right) ;
$$

similarly, equation (3.6) is equivalent to the statement

$$
u_{j, S k}=(-1)^{j+1} \mathrm{e}^{-i a \bullet S k} S u_{j, k} \quad \text { for } k \in \mathbf{Z}^{3},(S, a) \in \mathcal{H}^{-}\left(u_{0}\right) .
$$

In typical applications of the recursion scheme (3.2), where $u_{0}$ is a Fourier polynomial as well as its iterates $u_{j}$, equations (3.10), (3.11) can be used to speed up the computation of the Fourier components of the $u_{j}$ 's; in fact, at any given order $j$, after computing a Fourier component $u_{j, k}$ we immediately obtain from the cited equations the components $u_{j, S k}$ for all $S$ in the reduced subgroup or subspace $\mathcal{H}_{R}\left(u_{0}\right), \mathcal{H}_{R}^{-}\left(u_{0}\right)$.

Convergence of the power series in $\mathcal{A}$. From now on

$$
\tau:=\text { convergence radius of the series } \sum_{j=0}^{\infty} u_{j} t^{j} \text { in } \mathcal{A} .
$$

Furthermore,

$$
u: t \in(-\mathcal{T}, T) \mapsto u(t) \text { is the maximal } \mathcal{A} \text {-solution of the Cauchy problem }
$$

(recall that, for any $n>5 / 2, u$ is also the maximal $H^{n}$-solution). We note that

$$
0<\tau \leqslant \mathcal{T} \wedge T, \quad u(t)=\sum_{j=0}^{\infty} u_{j} t^{j} \quad \text { in } \mathcal{A}, \text { for } t \in(-\tau, \tau)
$$

(with $\wedge$ indicating the minimum). In fact: being analytic, $u$ admits a power series representation in a neighborhood of zero; this necessarily coincides with the series (3.1), whose convergence radius $\tau$ is thus nonzero and fulfills $(-\tau, \tau) \subset(-\mathcal{T}, T)$. 
Convergence of the power series in $\boldsymbol{H}^{n}$. After fixing $n \in[0,+\infty)$, let us discuss the series (3.1) in the Sobolev space $H^{n}$. To this purpose, we put

$$
\tau_{n}:=\text { convergence radius of the series } \sum_{j=0}^{\infty} u_{j} t^{j} \text { in } H^{n} ;
$$

the root test gives

$$
\tau_{n}=\liminf _{j \rightarrow+\infty}\left\|u_{j}\right\|_{n}^{-1 / j}
$$

(defining $\left.0^{-1 / j}:=+\infty\right)$. With $\tau, \mathcal{T}, T, u$ as before, we claim that

$$
\tau \leqslant \tau_{n} \text { and } u(t)=\sum_{j=0}^{\infty} u_{j} t^{j} \text { in } H^{n}, \text { for } t \in\left(-\mathcal{T} \wedge \tau_{n}, T \wedge \tau_{n}\right)
$$

(where $-\mathcal{T} \wedge \tau_{n}$ is the opposite of the minimum $\mathcal{T} \wedge \tau_{n}$ ). In fact: the series $\sum_{j=0}^{\infty} u_{j} t^{j}$ converges to $u(t)$ in $\mathcal{A}$, for $t \in(-\tau, \tau)$; by the continuous embedding $\mathcal{A} \hookrightarrow H^{n}$ this series converges to $u(t)$ in $H^{n}$ as well, at least for $t \in(-\tau, \tau)$; thus $\tau \leqslant \tau_{n}$. Moreover the functions $u:(-\mathcal{T}, T) \rightarrow H^{n}$ and $t \in\left(-\tau_{n}, \tau_{n}\right) \mapsto \sum_{j=0}^{\infty} u_{j} t^{j} \in H^{n}$ are analytic and coincide on $(-\tau, \tau)$; so, by the analytic continuation principle, these functions coincide on the intersection of their domains which is $\left(-\mathcal{T} \wedge \tau_{n}, T \wedge \tau_{n}\right)$. Let as add a stronger claim:

$$
\text { if } n>\frac{5}{2}, \quad \tau \leqslant \tau_{n} \leqslant \mathcal{T} \wedge T \text { and } u(t)=\sum_{j=0}^{\infty} u_{j} t^{j} \text { in } H^{n} \text {, for } t \in\left(-\tau_{n}, \tau_{n}\right) \text {. }
$$

In fact, the function $t \in\left(-\tau_{n}, \tau_{n}\right) \mapsto \sum_{j=0}^{\infty} u_{j} t^{j}$ is in $C\left(\left(-\tau_{n}, \tau_{n}\right), H^{n}\right) \cap C^{1}\left(\left(-\tau_{n}, \tau_{n}\right), H^{n-1}\right)$ and solves the Euler Cauchy problem, so it is a restriction of the maximal $H^{n}$-solution, which is $u$ of domain $(-\mathcal{T}, T)$; this gives the relations $\tau_{n} \leqslant \mathcal{T} \wedge T$ and $u(t)=\sum_{j=0}^{\infty} u_{j} t^{j}$ in $H^{n}$, for $t \in\left(-\tau_{n}, \tau_{n}\right)$.

Power series for the Sobolev norms of the solution. Let us choose $n \in[0,+\infty)$. The squared norm $\left\|\sum_{j=0}^{+\infty} u_{j} t^{j}\right\|_{n}^{2}=\left\langle\sum_{j=0}^{+\infty} u_{j} t^{j} \mid \sum_{j=0}^{+\infty} u_{j} t^{j}\right\rangle_{n}$ has the formal expansion

$$
\left\|\sum_{j=0}^{+\infty} u_{j} t^{j}\right\|_{n}^{2}=\sum_{j=0}^{+\infty} \nu_{n j} t^{j}, \quad \nu_{n j}:=\sum_{\ell=0}^{j}\left\langle u_{\ell} \mid u_{j-\ell}\right\rangle_{n} \in \mathbf{R}
$$

for future use we remark that $\left(^{7}\right)$

$$
\mathcal{H}^{-}\left(u_{0}\right) \neq \emptyset \Rightarrow \nu_{n j}=0 \text { for all } j \text { odd }
$$

Independently of any assumption on $\mathcal{H}^{-}\left(u_{0}\right)$, let us define

$$
\theta_{n}:=\text { convergence radius of the series } \sum_{j=0}^{\infty} \nu_{n j} t^{j}=\liminf _{j \rightarrow+\infty}\left|\nu_{n j}\right|^{-1 / j}
$$

Let us relate these objects to the convergence radius $\tau_{n}$ in $(3.15)$, to the solution $u \in C^{\omega}((-\mathcal{T}, T), \mathcal{A})$ and to its squared $H^{n}$ norm. We claim that

$$
\tau_{n} \leqslant \theta_{n} \text { and }\|u(t)\|_{n}^{2}=\sum_{j=0}^{+\infty} \nu_{n j} t^{j} \text { for } t \in\left(-\mathcal{T} \wedge \theta_{n}, T \wedge \theta_{n}\right)
$$

\footnotetext{
${ }^{7}$ Let us propose a proof of $(3.20)$, based directly on the definition $(3.19)$ of $\nu_{n j}$. If $\mathcal{H}^{-}\left(u_{0}\right)$ has at least one element $(S, a)$, from (3.6) and from the invariance of $\langle\mid\rangle_{n}$ under any transformation $\mathcal{E}_{*}(S, a)$ we obtain that, for each $\ell \in\{0, \ldots, j\},\left\langle u_{\ell} \mid u_{j-\ell}\right\rangle_{n}=$ $\left\langle(-1)^{\ell} \mathcal{E}_{*}(S, a) u_{\ell} \mid(-1)^{j-\ell} \mathcal{E}_{*}(S, a) u_{j-\ell}\right\rangle_{n}=(-1)^{j}\left\langle u_{\ell} \mid u_{j-\ell}\right\rangle_{n}$, whence $\nu_{n j}=(-1)^{j} \nu_{n j}$. If $j$ is odd, this means $\nu_{n j}=0$.
} 
(with $-\mathcal{T} \wedge \theta_{n}$ the opposite of $\mathcal{T} \wedge \theta_{n}$ ). In fact: the expansion $u(t)=\sum_{j=0}^{+\infty} u_{j} t^{j}$, converging in $H^{n}$ for $t \in\left(-\tau_{n}, \tau_{n}\right)$, implies $\tau_{n} \leqslant \theta_{n}$ and $\|u(t)\|_{n}^{2}=\sum_{j=0}^{+\infty} \nu_{n j} t^{j}$ for $t \in\left(-\tau_{n}, \tau_{n}\right)$. Moreover the functions $t \in(-\mathcal{T}, T) \mapsto\|u(t)\|_{n}^{2}$ and $t \in\left(-\theta_{n}, \theta_{n}\right) \mapsto \sum_{j=0}^{+\infty} \nu_{n j} t^{j}$ are analytic and coincide on $\left(-\tau_{n}, \tau_{n}\right)$, so they coincide everywhere on the intersections of their domains, which is $\left(-\mathcal{T} \wedge \theta_{n}, T \wedge \theta_{n}\right)$. We now add to (3.22) a stronger claim:

$$
\text { if } n>\frac{5}{2}, \quad \tau_{n} \leqslant \theta_{n} \leqslant \mathcal{T} \wedge T \text { and }\|u(t)\|_{n}^{2}=\sum_{j=0}^{+\infty} \nu_{n j} t^{j} \text { for } t \in\left(-\theta_{n}, \theta_{n}\right) \text {. }
$$

Let us prove this claim, assuming for example that $\mathcal{T} \wedge T=T$. If it were $T<\theta_{n}$ we would infer $\lim _{t \rightarrow T^{-}}\|u(t)\|_{n}^{2}=$ $\lim _{t \rightarrow T^{-}} \sum_{j=0}^{+\infty} \nu_{n j} t^{j}=\sum_{j=0}^{+\infty} \nu_{n j} T^{j}<+\infty$ (the first equality would hold due to (3.22) and $T \wedge \theta_{n}=T$; the subsequent two relations would hold because $T$ would be inside the convergence interval of the series). On the other hand, since $n>5 / 2$, the existence and finiteness of $\lim _{t \rightarrow T^{-}}\|u(t)\|_{n}^{2}$ would contradict (2.15).

\section{Power series for the Euler equation in a paper of Behr, NeČas and Wu}

In the paper [6] mentioned above, the authors considered the power series (3.1) for the Euler equation on $\mathbf{T}^{3}$, with an initial datum $u_{0} \in \mathcal{A}$ given by

$$
\begin{gathered}
u_{0}(x)=\sum_{k= \pm a, \pm b, \pm c} u_{0 k} \mathrm{e}^{i k \bullet x}, \\
a:=(1,1,0), \quad b:=(1,0,1), \quad c:=(0,1,1) ; \\
u_{0, \pm a}:=(1,-1,0), \quad u_{0, \pm b}:=(1,0,-1), \quad u_{0, \pm c}:=(0,1,-1) .
\end{gathered}
$$

Like $u_{0}$, all the subsequent terms $u_{j}$ are Fourier polynomials with rational coefficients $\left({ }^{8}\right)$. Using rules equivalent to $(3.2),(2.5)$, the terms $u_{j}$ were determined in [6] by computer algebra, for $j=1,2, \ldots, 35$. Computations were done with Mathematica for $j=1, \ldots, 10$, and with a $C_{++}$program for $j=11, \ldots, 35$ (in the later case, approximating the rational coefficients with finite precision decimal numbers). After determining the $u_{j}$ 's, the authors fixed their attention on the partial sums

$$
u^{(N)}(t):=\sum_{j=0}^{N} u_{j} t^{j}
$$

whose $N \rightarrow+\infty$ limit gives the solution $u(t)$ of the Euler Cauchy problem, for all $t$ such that the series converges. The previously mentioned computation of the $u_{j}$ 's made available these partial sums for $N=0,1, \ldots, 35$; the authors of [6] computed the (squared) Sobolev norm

$$
\left\|u^{(N)}(t)\right\|_{3}^{2}=\sum_{k \in \mathbf{Z}^{3}}|k|^{6}\left|u_{k}^{(N)}(t)\right|^{2}
$$

for the above values of $N$, and several values of $t$. Their main results were the following:

(i) Setting $t=0.32$, and analyzing the behavior of $\left\|u^{(N)}(0.32)\right\|_{3}$ for $N$ from 0 to 35 , the authors found evidence that $\left\|u^{(N)}(0.32)\right\|_{3}$ should approach a finite limit for $N \rightarrow+\infty$.

(ii) Setting $t=0.35$, the authors observed a rapid growth of $\left\|u^{(N)}(0.35)\right\|_{3}$ for $N$ ranging from 0 to 35, a fact suggesting that $\lim _{N \rightarrow+\infty}\left\|u^{(N)}(0.35)\right\|_{3}=+\infty$.

(iii) A behavior as in (ii) was found to occur for slightly higher values of $t$ (even though the authors suspected some rounding error to appear for $t>0.35)$.

\footnotetext{
${ }^{8}$ For a more precise statement on these cofficients see our discussion of the datum $u_{0}$ in the next section and, in particular, equation (5.9).
} 
The above results suggest that the series $\sum_{j=0}^{+\infty} u_{j} t^{j}$ has a finite convergence radius $\tau_{3}$ in $H^{3}$, with $\tau_{3} \in$ $(0.32,0.35)$.

Let us discuss this outcome from the viewpoint of the present paper, denoting with $u$ the maximal $\mathcal{A}$-solution of the Cauchy problem with this datum and recalling that this coincides with the maximal $H^{3}$-solution. The datum $u_{0}$ possesses pseudo-symmetries (to be described in the next section); therefore, $u$ has a time symmetric domain $(-T, T)$ (in [6] this fact was not explicitly declared, but probably regarded as self-evident). According to our equation (3.18), it is

$$
\tau_{3} \leqslant T
$$

in principle, it could be $T=+\infty$. In spite of this, the authors of [6] spoke of a blow-up at $\tau_{3}$.

In the next two sections we present our computations on the power series for the Behr-Nečas-Wu initial datum, with our interpretation of the results. Even though these calculations confirm the "experimental" outcomes (i)-(iii) of [6], we give evidence that the solution $u$ of the Euler equation does not blow up close to $\tau_{3}$; on the contrary, computing the power series for $\|u(t)\|_{3}^{2}$ up the available order we obtain strong evidence that such a power series has a convergence radius $\theta_{3}$ such that $0.47<\theta_{3}<0.50$, which implies for the time $T$ of existence of $u$ the bound $T \geqslant \theta_{3}>0.47$. By a subsequent analysis relying on the technique of the Padé approximants, we show that a blow-up of $u(t)$ might happen at a time larger than 0.48: more precisely, these computations give a somehow weak indication that $T$ might be finite, with $0.56<T<0.73$.

\section{Our approach to the power series of Behr, NeČas and Wu}

Let us denote again with $u_{0}$ the datum (4.1) and consider its iterates $u_{j}(j=1,2, \ldots)$, with the corresponding power series; like $u_{0}$, all the iterates $u_{j}$ are Fourier polynomials with rational coefficients. Throughout the section, $u$ is the maximal $\mathcal{A}$-solution of the Euler equation with datum $u_{0}$.

A closer analysis of the Behr-Nečas-Wu initial datum: symmetry properties. The symmetry group $\mathcal{H}\left(u_{0}\right)$ and the pseudo-symmetry space $\mathcal{H}^{-}\left(u_{0}\right)$ (Eqs. $\left.(2.34),(2.35)\right)$ can be explicitly computed. For the first one, we find

$$
\begin{gathered}
\mathcal{H}\left(u_{0}\right)=\left\{(\mathbf{1}, 0),\left(\mathbf{1}, \imath_{2}\right),\left(A, a_{1}\right),\left(A, a_{2}\right),\left(B, a_{1}\right),\left(B, a_{2}\right),\right. \\
\left.\left(C, c_{1}\right),\left(C, c_{2}\right),\left(D, c_{1}\right),\left(D, c_{2}\right),(E, 0),\left(E, \imath_{2}\right)\right\}
\end{gathered}
$$

where 1 is the $3 \times 3$ identity matrix, and

$$
\begin{gathered}
A:=\left(\begin{array}{lll}
0 & 1 & 0 \\
0 & 0 & 1 \\
1 & 0 & 0
\end{array}\right), \quad B:=\left(\begin{array}{ccc}
0 & -1 & 0 \\
-1 & 0 & 0 \\
0 & 0 & -1
\end{array}\right) \\
C:=\left(\begin{array}{lll}
0 & 0 & 1 \\
1 & 0 & 0 \\
0 & 1 & 0
\end{array}\right), \quad D:=\left(\begin{array}{ccc}
-1 & 0 & 0 \\
0 & 0 & -1 \\
0 & -1 & 0
\end{array}\right) \quad E:=\left(\begin{array}{ccc}
0 & 0 & -1 \\
0 & -1 & 0 \\
-1 & 0 & 0
\end{array}\right) ;
\end{gathered}
$$

furthermore, $\imath_{2}, a_{1}$, etc., are the following elements of $\mathbf{T}^{3}$ :

$$
\begin{gathered}
\imath_{2}:=(\pi, \pi, \pi), \quad a_{1}:=(0,0, \pi), \quad a_{2}:=(\pi, \pi, 0), \\
c_{1}:=(\pi, 0,0), \quad c_{2}:=(0, \pi, \pi)
\end{gathered}
$$

(of course, in the above $\pi$ is short for $\pi \bmod .2 \pi \mathbf{Z}$ ). Let us fix the attention on the reduced symmetry subgroup $\mathcal{H}_{R}\left(u_{0}\right)=\{\mathbf{1}, A, B, C, D, E\} ;$ it is readily checked that

$$
\begin{gathered}
A^{3}=\mathbf{1}, B^{2}=\mathbf{1},(B A)^{2}=1 \\
C=A^{2}, \quad D=A B, \quad E=A^{2} B .
\end{gathered}
$$


So, $\mathcal{H}_{R}\left(u_{0}\right)$ has two generators $A, B$; the first line in (5.4) gives a presentation of this group in terms of generators and relations, while the second line expresses the other elements in terms of $A, B$. Using equation (5.4), one recognizes a group isomorphism

$$
\mathcal{H}_{R}\left(u_{0}\right) \simeq \mathbf{D}_{3}
$$

where the right-hand side indicates the dihedral group of order 3 , formed by the symmetries of an equilateral triangle $\left({ }^{9}\right)$.

Now we consider the full group $\mathcal{H}\left(u_{0}\right)$ (with the product (2.22)). It is easy to check that

$$
\begin{gathered}
\left(A, a_{1}\right)^{6}=(\mathbf{1}, 0), \quad\left(B, a_{1}\right)^{2}=(\mathbf{1}, 0),\left(\left(B, a_{1}\right)\left(A, a_{1}\right)\right)^{2}=(\mathbf{1}, 0), \\
\left(A, a_{1}\right)^{2}=\left(C, c_{2}\right), \quad\left(A, a_{1}\right)^{3}=\left(\mathbf{1}, \imath_{2}\right), \quad\left(A, a_{1}\right)^{4}=\left(A, a_{2}\right), \quad\left(A, a_{1}\right)^{5}=\left(C, c_{1}\right), \\
\left(A, a_{1}\right)\left(B, a_{1}\right)=\left(D, c_{2}\right), \quad\left(A, a_{1}\right)^{2}\left(B, a_{1}\right)=\left(E, \imath_{2}\right), \quad\left(A, a_{1}\right)^{3}\left(B, a_{1}\right)=\left(B, a_{2}\right), \\
\left(A, a_{1}\right)^{4}\left(B, a_{1}\right)=\left(D, c_{1}\right), \quad\left(A, a_{1}\right)^{5}\left(B, a_{1}\right)=(E, 0) .
\end{gathered}
$$

So, $\mathcal{H}\left(u_{0}\right)$ has two generators $\left(A, a_{1}\right)$ and $\left(B, a_{1}\right)$; the first line in (5.6) gives a presentation of this group in terms of generators and relations, and the subsequent lines express the other elements in terms of the generators. One recognizes a group isomorphism

$$
\mathcal{H}\left(u_{0}\right) \simeq \mathbf{D}_{6}
$$

where the right-hand side indicates the dihedral group of order 6 , formed by the symmetries of a hexagon (see the previous footnote).

Let us pass to the pseudo-symmetry space $\mathcal{H}^{-}\left(u_{0}\right)$. One readily checks that this contains $(-\mathbf{1}, 0)$ (inducing the space reflection $\left.\mathcal{E}(-\mathbf{1}, 0): x \in \mathbf{T}^{3} \mapsto-x\right)$. From here and from the general result $(2.40)$, one obtains

$$
\begin{gathered}
\mathcal{H}^{-}\left(u_{0}\right)=\mathcal{H}\left(u_{0}\right)(-\mathbf{1}, 0)=\left\{(-\mathbf{1}, 0),\left(-\mathbf{1}, \imath_{2}\right),\left(-A, a_{1}\right),\left(-A, a_{2}\right),\right. \\
\left.\left(-B, a_{1}\right),\left(-B, a_{2}\right),\left(-C, c_{1}\right),\left(-C, c_{2}\right),\left(-D, c_{1}\right),\left(-D, c_{2}\right),(-E, 0),\left(-E, \imath_{2}\right)\right\},
\end{gathered}
$$

with $A, B, \ldots$ and $\imath_{2}, a_{1}, \ldots$ as in equations (5.2)-(5.3).

\section{Some consequences of the previous symmetry results.}

(i) What we have stated in Section 3 for an arbitrary initial datum holds, in particular, for the present datum $u_{0}$ : the symmetries or pseudo-symmetries of $u_{0}$ can be used to speed up the computation of the Fourier components of any iterate $u_{j}$. More precisely, if we know the Fourier component $u_{j, k}$ for some $k$, using equations (3.10), (3.11) we readily obtain the components $u_{j, S k}$ for all $S \in \mathcal{H}_{R}\left(u_{0}\right) \cup \mathcal{H}_{R}^{-}\left(u_{0}\right)$.

(ii) As already noted, the pseudo-symmetry space $\mathcal{H}^{-}\left(u_{0}\right)$ contains $(-\mathbf{1}, 0)$, corresponding to the space reflection. In terms of Fourier coefficients, the relation (3.11) with $(S, a)=(-\mathbf{1}, 0)$ takes the form $u_{j,-k}=(-1)^{j} u_{j, k}$ for $j=0,1,2, \ldots$ and $k \in \mathbf{Z}^{3}$. On the other hand, any iterate $u_{j}$ is a real vector field, thus $u_{j,-k}=\overline{u_{j, k}}$; in conclusion $\overline{u_{j, k}}=(-1)^{j} u_{j, k}$, which indicates that $u_{j, k}$ is real for $j$ even, and imaginary for $j$ odd. Taking into account that the coefficients $u_{j, k}$ are rational in any case, we conclude the following for each $k \in \mathbf{Z}^{3}$ :

$$
u_{j, k} \in \mathbf{Q}^{3} \text { for } j=0,2,4, \ldots ; \quad u_{j, k} \in i \mathbf{Q}^{3} \text { for } j=1,3,5, \ldots
$$

(iii) In the sequel we are often interested in the partial sums $u^{(N)}(t):=\sum_{j=0}^{N} u_{j} t^{j}$ and in their norms $\left\|u^{(N)}(t)\right\|_{n}$, especially for $n=3$. Since $\mathcal{H}^{-}\left(u_{0}\right) \neq \emptyset$, as in (3.9) we have $\left\|u_{N}(t)\right\|_{n}=\left\|u_{N}(-t)\right\|_{n}$.

\footnotetext{
${ }^{9}$ For any integer $n \in\{3,4, \ldots\}$, one denotes with $\mathbf{D}_{n}$ the dihedral group of order $n$; this is formed by the orthogonal transformations of the Euclidean plane $\mathbf{R}^{2}$ into itself which preserve a regular polygon with $n$ sides, centered at the origin. Denoting with $i d$ the identity map, with $a$ the rotation of an angle $2 \pi / n$ and with $b$ the reflection about anyone of the $n$ symmetry axes of the polygon, one finds that $a, b$ are generators of $\mathbf{D}_{n}$ and fulfill the relations $a^{n}=i d, b^{2}=i d,(b a)^{2}=i d$. The elements of $\mathbf{D}_{n}$ are $2 n$, and coincide with $i d, a, a^{2}, \ldots, a^{n-1}, b, a b, a^{2} b, \ldots, a^{n-1} b$.
} 
(iv) Independently of any convergence consideration about the power series $\sum_{j=0}^{+\infty} u_{j} t^{j}$, the result $\mathcal{H}^{-}\left(u_{0}\right) \neq \emptyset$ also ensures that the (maximal $\mathcal{A}$-) solution $u$ of the Euler equation with datum $u_{0}$ has a symmetric domain $(-T, T)$ (recall Eq. $(2.32))$.

Describing our computations. We have considered again the power series (3.1) for the datum $u_{0}$; to deal with this series we have written a program in Python, using the package gmpy [26] for fast arithmetics on rational numbers. This program implements equation (2.5) for $\mathcal{P}$ and the recursion rule (3.2); moreover, it takes into account the dihedral symmetries (and pseudosymmetries) of $u_{0}$ to speed up computations. The program has been run to compute the terms $u_{j}$ for $j=1, \ldots, 52\left({ }^{10}\right)$. Calculations have been performed on a PC with an Intel Core i7 CPU 860 at 2.8GHz and an 8GB RAM. The CPU time for $u_{j}$ has been, for example: 1 second for $j=10$, one minute for $j=20$, half an hour for $j=30,7 \mathrm{~h}$ for $j=40$ and $85 \mathrm{~h}$ for $j=52$. Differently from [6], for all orders up to $j=52$ the Fourier coefficients $u_{j, k}$ of $u_{j}$ have been represented as elements of $\mathbf{Q}^{3}$ or $i \mathbf{Q}^{3}$; so, no rounding errors related to finite precision arithmetics have been introduced in the calculation of the power series.

From the $u_{j}$ 's one determines the squared norms $\left\|u_{j}\right\|_{3}^{2}=\sum_{k \in \mathbf{Z}^{3}}|k|^{6}\left|u_{j, k}\right|^{2}$, the partial sums $u^{(N)}(t):=$ $\sum_{j=0}^{N} u_{j} t^{j}$ and their squared norms $\left\|u^{(N)}(t)\right\|_{3}^{2}=\sum_{k \in \mathbf{Z}^{3}}|k|^{6}\left|u_{k}^{(N)}(t)\right|^{2}(N=1, \ldots, 52)$. Each $\left\|u_{j}\right\|_{3}^{2}$ is a rational number and $\left\|u^{(N)}(t)\right\|_{3}^{2}$ is a polynomial of order $2 N$ in $t$, with rational coefficients, containing only even powers of $t$; furthermore, the coefficients of $t^{0}$ and $t^{2 N}$ in $\left\|u^{(N)}(t)\right\|_{3}^{2}$ are $\left\|u_{0}\right\|_{3}^{2}$ and $\left\|u_{N}\right\|_{3}^{2}$, respectively.

Our computations of the above norms, up to $j=52$ or $N=52$, have been done using the previously mentioned Python program. These calculations have been relatively quick: for example, the computation of $\left\|u^{(52)}(t)\right\|_{3}^{2}$ has required a $\mathrm{CPU}$ time of about $3 \mathrm{~h}$. As first examples of our results, we report the following ones:

$$
\begin{gathered}
\left\|u_{0}\right\|_{3}^{2}=96, \quad\left\|u_{1}\right\|_{3}^{2}=6912, \quad\left\|u_{2}\right\|_{3}^{2}=45440 \\
\left\|u_{3}\right\|_{3}^{2}=\frac{3695360}{9}, \quad\left\|u_{4}\right\|_{3}^{2}=\frac{1366793248}{675}, \quad\left\|u_{5}\right\|_{3}^{2}=\frac{2243123779689032}{186046875} .
\end{gathered}
$$

$\left\|u_{52}\right\|_{3}^{2}$ is a ratio of integers where the numerator and the denominator have 19515 and 19463 digits, respectively. Table 1 reports $\left\|u_{j}\right\|_{3}^{2}$ for $j=0, \ldots, 52$, in the 16 digits decimal representation.

Let us pass to the squared norms $\left\|u^{(N)}(t)\right\|_{3}^{2}$. As an example, the result for $N=5$ is

$$
\begin{gathered}
\left\|u^{(5)}(t)\right\|_{3}^{2}=96+6656 t^{2}+\frac{258304}{9} t^{4}+\frac{104566912}{525} t^{6} \\
-\frac{9513575648}{70875} t^{8}+\frac{2243123779689032}{186046875} t^{10} .
\end{gathered}
$$

There is no room to report here the results obtained for all the other values of $N$, especially in the rational form for the coefficients. However, we can write some of them in the 16 digits precision; in particular,

$$
\begin{gathered}
\left\|u^{(52)}(t)\right\|_{3}^{2}=96+6656 t^{2}+2.870044444444444 \times 10^{4} t^{4}+1.993359937918871 \times 10^{5} t^{6} \\
+1.058054454761424 \times 10^{5} t^{8}+1.781444415306641 \times 10^{6} t^{10}+2.740017914111055 \times 10^{6} t^{12} \\
-7.321985472578865 \times 10^{6} t^{14}+4.183410651491110 \times 10^{6} t^{16}+1.457483700816015 \times 10^{8} t^{18} \\
-1.768517246168822 \times 10^{8} t^{20}+4.196205149715839 \times 10^{8} t^{22}+3.648789154816725 \times 10^{9} t^{24} \\
-2.178830191383206 \times 10^{10} t^{26}-1.394064522752687 \times 10^{10} t^{28}+2.954202883502504 \times 10^{11} t^{30}
\end{gathered}
$$

\footnotetext{
${ }^{10}$ To test the reliability of this program, the calculation of some of the $u_{j}^{\prime} s$ has been checked in two independent ways. These checks have been done by means of other two programs, which implement equations (2.5)-(3.2) accepting as an initial datum $u_{0}$ any Fourier polynomial; these do not refer to any symmetry property of $u_{0}$. The first of these programs, written in Mathematica, has been used to compute the $u_{j}$ 's up to order $j=13$; the second program, written in Python, has been used for a calculation up to $j=43$.
} 


$$
\begin{aligned}
& +1.283692616423054 \times 10^{11} t^{32}-4.543575106022102 \times 10^{12} t^{34}+4.789569007452901 \times 10^{12} t^{36} \\
& +2.830635227431622 \times 10^{13} t^{38}+4.470168139346678 \times 10^{13} t^{40}-6.910532995061547 \times 10^{14} t^{42} \\
& +1.457019276470951 \times 10^{14} t^{44}+9.053007124662626 \times 10^{15} t^{46}-8.939780851014422 \times 10^{15} t^{48} \\
& -1.019952729404346 \times 10^{17} t^{50}+1.137772938577812 \times 10^{17} t^{52}+1.644161010427522 \times 10^{18} t^{54} \\
& -4.571936581656874 \times 10^{18} t^{56}-3.140936865806385 \times 10^{19} t^{58}+2.408085513008218 \times 10^{21} t^{60} \\
& -1.107900217253947 \times 10^{23} t^{62}+4.186064092726056 \times 10^{24} t^{64}-1.674853723772203 \times 10^{26} t^{66} \\
& +6.911508987260593 \times 10^{27} t^{68}-2.698282390313396 \times 10^{29} t^{70}+9.951375797771149 \times 10^{30} t^{72} \\
& -3.558771163372845 \times 10^{32} t^{74}+1.232107326257251 \times 10^{34} t^{76}-4.045044388392564 \times 10^{35} t^{78} \\
& +1.242344004413423 \times 10^{37} t^{80}-3.561397641466941 \times 10^{38} t^{82}+9.520206481050174 \times 10^{39} t^{84} \\
& -2.357932432543021 \times 10^{41} t^{86}+5.354229494719748 \times 10^{42} t^{88}-1.103667607665446 \times 10^{44} t^{90} \\
& +2.052382635232918 \times 10^{45} t^{92}-3.436006560519912 \times 10^{46} t^{94}+5.184487278969682 \times 10^{47} t^{96} \\
& -7.072466985323957 \times 10^{48} t^{98}+8.759614973466463 \times 10^{49} t^{100}-9.896987665647683 \times 10^{50} t^{102} \\
& +1.025058601409640 \times 10^{52} t^{104}
\end{aligned}
$$

The rest of the paper reports a number of facts stemming from our computations, with the interpretation that we suggest for them.

Verification of the outcomes of [6] on $\left\|u^{(N)}(t)\right\|_{3}^{2}$. Our computations based on the systematic use of rational numbers have given essentially the same results as in [6] about $\left\|u^{(N)}(t)\right\|_{3}^{2}$ as a function of $N$, in the two cases $t=0.32$ and $t=0.35$. So, $\left\|u^{(N)}(0.32)\right\|_{3}^{2}$ seems to approach a limit value for large $N$, while $\left\|u^{(N)}(0.35)\right\|_{3}^{2}$ grows rapidly with $N$; our use of rational coefficients ensures that such a rapid growth is not due to cumulative rounding errors. In Figures 1-2, we report $\left\|u^{(N)}(t)\right\|_{3}^{2}$ as a function of $N \in\{0, \ldots, 52\}$, in the two cases $t=0.32$ and $t=0.35$; these figures are very similar to the ones at the bottom of pages 235 and 236 of [6], respectively (but comparison requires a rescaling, since the $H^{3}$ norm employed in [6] differs from ours by a constant factor).

We agree with [6] in interpreting these results as indications that the power series for this initial datum has a finite $H^{3}$-convergence radius $\tau_{3}$, with $\tau_{3} \in(0.32,0.35)$.

Further evidence on the $\boldsymbol{H}^{3}$-convergence radius of the power series. This comes from the root test (3.16) for $n=3$ :

$$
\tau_{3}=\liminf _{j \rightarrow+\infty}\left\|u_{j}\right\|_{3}^{-1 / j} .
$$

Figure 3 represents $\left\|u_{j}\right\|_{3}^{-1 / j}$ as a function of $j \in\{1, \ldots, 52\}$. For $j=36,38, \ldots, 52$ we have a very good interpolation

$$
\left\|u_{j}\right\|_{3}^{-1 / j} \simeq 0.32158-\left(\frac{1.20125}{j}\right)^{1.38458},
$$

(obtained assuming for the interpolant the form $A-(B / j)^{c}$, and applying the least squares criterion).

The right-hand side of (5.14) approximates $\left\|u_{j}\right\|_{3}^{-1 / j}$ with a mean quadratic error $<10^{-5}$ (averaging, as indicated, for $j=36,38, \ldots, 52$; if we average over the larger range $j=16,18, \ldots, 52$, the mean quadratic error is $\left.<10^{-4}\right)$. Assuming that (5.14) approximates $\left\|u_{j}\right\|_{3}^{-1 / j}$ with a similar precision for arbitrarily large $j$, but keeping cautiously only two digits in our final guess we conclude with an estimate

$$
0.32<\tau_{3}<0.33
$$


TABLE 1 . The squared norms $\left\|u_{j}\right\|_{3}^{2}$.

\begin{tabular}{|l|l||l|l|}
\hline$j$ & $\left\|u_{j}\right\|_{3}^{2}$ & $j$ & $\left\|u_{j}\right\|_{3}^{2}$ \\
\hline 1 & 6912 & 27 & $4.070323867244879 \times 10^{27}$ \\
2 & 45440 & 28 & $3.800202819232687 \times 10^{28}$ \\
3 & $4.105955555555556 \times 10^{5}$ & 29 & $3.554589555246873 \times 10^{29}$ \\
4 & $2.024878885925926 \times 10^{6}$ & 30 & $3.330557264153261 \times 10^{30}$ \\
5 & $1.205676676745595 \times 10^{7}$ & 31 & $3.125627141295364 \times 10^{31}$ \\
6 & $8.452219877103332 \times 10^{7}$ & 32 & $2.937654907691943 \times 10^{32}$ \\
7 & $6.152775603322622 \times 10^{8}$ & 33 & $2.764771414352126 \times 10^{33}$ \\
8 & $4.791192836997696 \times 10^{9}$ & 34 & $2.605347861791808 \times 10^{34}$ \\
9 & $3.628869598772102 \times 10^{10}$ & 35 & $2.457968790658826 \times 10^{35}$ \\
10 & $2.825486371143428 \times 10^{11}$ & 36 & $2.321406184470901 \times 10^{36}$ \\
11 & $2.228507964437443 \times 10^{12}$ & 37 & $2.194593722846032 \times 10^{37}$ \\
12 & $1.821213808657725 \times 10^{13}$ & 38 & $2.076602420620089 \times 10^{38}$ \\
13 & $1.539790191793044 \times 10^{14}$ & 39 & $1.966618988613002 \times 10^{39}$ \\
14 & $1.341372343677860 \times 10^{15}$ & 40 & $1.863927582086700 \times 10^{40}$ \\
15 & $1.190159209731028 \times 10^{16}$ \\
16 & $1.066432595016119 \times 10^{17}$ & 41 & $1.767894900465337 \times 10^{41}$ \\
17 & $9.598519025230687 \times 10^{17}$ & 42 & $1.677958174980847 \times 10^{42}$ \\
18 & $8.662788463495777 \times 10^{18}$ & 43 & $1.593615440091581 \times 10^{43}$ \\
44 & $1.514417532673484 \times 10^{44}$ \\
19 & $7.840631870939454 \times 10^{19}$ \\
20 & $7.122921654632158 \times 10^{20}$ \\
21 & $6.499436510134908 \times 10^{21}$ & 45 & $1.439961389630372 \times 10^{45}$ \\
22 & $5.957837347113741 \times 10^{22}$ & $1.369884345517744 \times 10^{46}$ \\
23 & $5.485035371335649 \times 10^{23}$ \\
24 & $5.068929708200902 \times 10^{24}$ & 47 & $1.303859232337703 \times 10^{47}$ \\
25 & $4.699401376031744 \times 10^{25}$ & $1.241590147950303 \times 10^{48}$ \\
26 & $4.368534165204974 \times 10^{26}$ & 49 & $1.182808795435820 \times 10^{49}$ \\
50 & $1.127271314453561 \times 10^{50}$ \\
51 & $1.074755536205362 \times 10^{51}$ \\
52 & $1.025058601409640 \times 10^{52}$ \\
\hline
\end{tabular}

Remainder estimates for the series expansion of $\boldsymbol{u}(\boldsymbol{t})$ in $\boldsymbol{H}^{3}$. Let $N \in\{0,1,2, \ldots\}$; of course

$$
u(t)-u^{(N)}(t)=\sum_{j=N+1}^{+\infty} u_{j} t^{j} \quad \text { for } t \in\left(-\tau_{3}, \tau_{3}\right)
$$

this implies

$$
\left\|u(t)-u^{(N)}(t)\right\|_{3} \leqslant \sum_{j=N+1}^{+\infty}\left\|u_{j}\right\|_{3}|t|^{j} \quad \text { for } t \in\left(-\tau_{3}, \tau_{3}\right) .
$$

To go on, we need a guess on the behavior of the norms $\left\|u_{j}\right\|_{3}$. To this purpose, let us consider the sequence

$$
\mu_{3 j}:=0.32^{j}\left\|u_{j}\right\|_{3} \quad(j=0,1,2 \ldots)
$$

recalling that 0.32 is the lower bound for $\tau_{3}$ in (5.15). From the norms available up to $j=52$, we can check that $\left(\mu_{3 j}\right)$ is decreasing while $j$ ranges in $\{1,2, \ldots, 52\}$; by extrapolation, let us assume that $\left(\mu_{3 j}\right)$ is decreasing on the infinite set $\{1,2, \ldots\}$. So, $\mu_{3 j} \leqslant \mu_{3 N}$ for integer $j \geqslant N \geqslant 1$, i.e.,

$$
\left\|u_{j}\right\|_{3} \leqslant \frac{\mu_{3 N}}{0.32^{j}} \quad \text { for } j \geqslant N \geqslant 1 .
$$




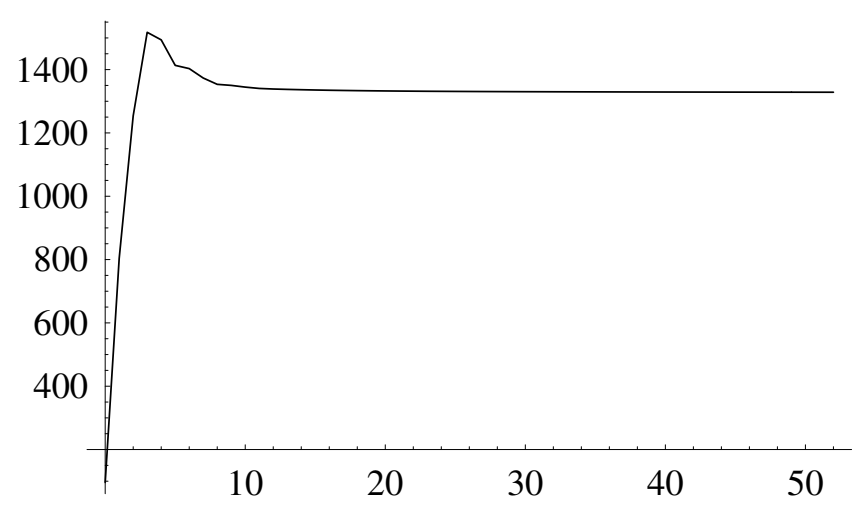

Figure 1. $\left\|u^{(N)}(0.32)\right\|_{3}^{2}$ as a function of $N \in\{0,1, \ldots, 52\}$.

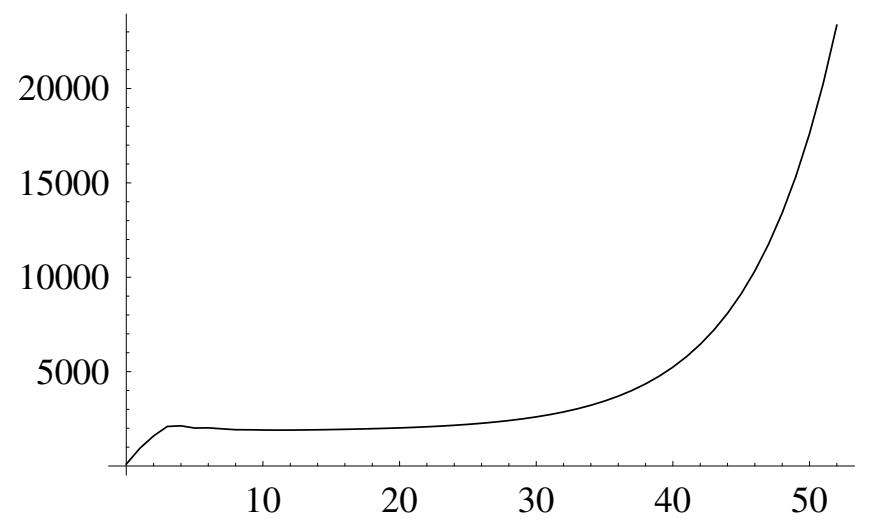

Figure 2. $\left\|u^{(N)}(0.35)\right\|_{3}^{2}$ as a function of $N \in\{0,1, \ldots, 52\}$.

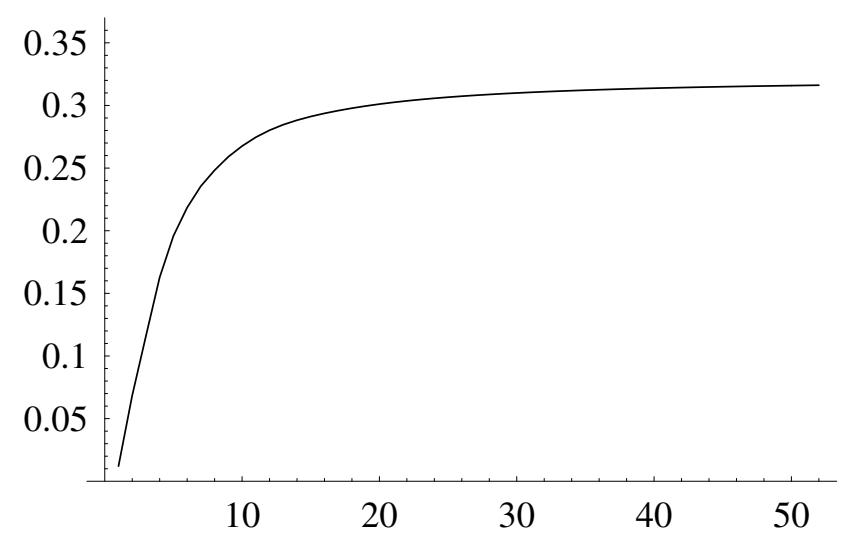

Figure $3 .\left\|u_{j}\right\|_{3}^{-1 / j}$ as a function of $j \in\{1, \ldots, 52\}$. 
For $t \in(-0.32,0.32)$, inserting this inequality into (5.17) we get $\left\|u(t)-u^{(N)}(t)\right\|_{3} \leqslant \mu_{3 N} \sum_{j=N+1}^{+\infty}|t / 0.32|^{j}=$ $\mu_{3 N}|t / 0.32|^{N+1} \sum_{j=0}^{+\infty}|t / 0.32|^{j}$, i.e.,

$$
\left\|u(t)-u^{(N)}(t)\right\|_{3} \leqslant \mu_{3 N} \frac{|t / 0.32|^{N+1}}{1-|t / 0.32|} \text { for } t \in(-0.32,0.32), N \in\{1,2,3, \ldots\} .
$$

Of course, this is a conjecture based on the previous extrapolation. For the practical application of the remainder estimate (5.20), we mention that (rounding up from above)

$$
\begin{aligned}
& \mu_{35}=11.7, \mu_{310}=5.99, \mu_{320}=3.39 \\
& \mu_{330}=2.61, \mu_{340}=2.20, \mu_{352}=1.88 .
\end{aligned}
$$

No blow-up at $\tau_{3}$. After accumulating indications that the Taylor series for $u(t)$ has an $H^{3}$-convergence radius $\tau_{3} \in(0.32,0.33)$, in the rest of the section we will present evidence that $u(t)$ does not blow up at $t=\tau_{3}$.

The power series for $\|u(t)\|_{3}^{2}$; an indication that $u(t)$ exists up to $t=0.47$ at least. The results (3.19)-(3.20) with $n=3$ give a formal series expansion

$$
\left\|\sum_{j=0}^{+\infty} u_{j} t^{j}\right\|_{3}^{2}=\sum_{j=0}^{+\infty} \nu_{3 j} t^{j}, \quad \nu_{3 j}:=\sum_{\ell=0}^{j}\left\langle u_{\ell} \mid u_{j-\ell}\right\rangle_{3} \in \mathbf{R}, \quad \nu_{3 j}=0 \quad \text { for } j \text { odd }
$$

the series $\sum_{j=0}^{+\infty} \nu_{3 j} t^{j}$ has a convergence radius

$$
\theta_{3}=\liminf _{j \rightarrow+\infty}\left|\nu_{3 j}\right|^{-1 / j}
$$

Recalling that $(-T, T)$ is the domain of the solution $u$, we know (from (3.23)) that

$$
\tau_{3} \leqslant \theta_{3} \leqslant T, \quad\|u(t)\|_{3}^{2}=\sum_{j=0}^{+\infty} \nu_{3 j} t^{j} \quad \text { for } t \in\left(-\theta_{3}, \theta_{3}\right) .
$$

In the sequel, for $N=0,1,2, \ldots$ we also consider the partial sums

$$
\nu_{3}^{(N)}(t):=\sum_{j=0}^{N} \nu_{3 j} t^{j}
$$

Of course, $u^{(N)}(t)=\sum_{j=0}^{N} u_{j} t^{j}$ is such that $u(t)=u^{(N)}(t)+O\left(t^{N+1}\right)$ for $t \rightarrow 0$; this implies $\|u(t)\|_{3}^{2}=$ $\left\|u^{(N)}(t)\right\|_{3}^{2}+O\left(t^{N+1}\right)$, whence

$$
\nu_{3}^{(N)}(t)=\left.\left\|u^{(N)}(t)\right\|_{3}^{2}\right|_{t^{k} \rightarrow 0 \text { for } k>N^{*}}
$$

With this remark, the previuos computations of $\left\|u^{(N)}(t)\right\|_{3}^{2}$ up to $N=52$ also give the partial sums $\nu_{3}^{(N)}(t)$ for $N \leqslant 52$ or, equivalently, the coefficients $\nu_{3 j}$ for $j \leqslant 52$. For example,

$$
\begin{gathered}
\nu_{30}=96, \quad \nu_{32}=6656, \quad \nu_{34}=\frac{258304}{9}, \quad \nu_{36}=\frac{2825587712}{14175}, \\
\nu_{38}=\frac{52545219363488}{496621125}, \quad \nu_{310}=\frac{10025320340466597351685768}{5627635784943046875} ;
\end{gathered}
$$

$\nu_{352}$ is a ratio of integers where the numerator and the denominator have 2610 and 2593 digits, respectively. 


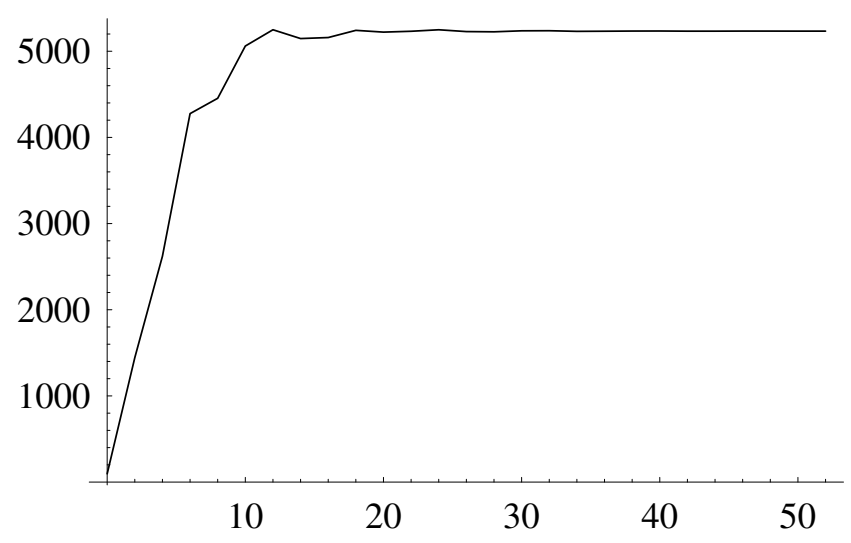

Figure 4. $\nu_{3}^{(N)}(0.45)$ as a function of $N \in\{0,2, \ldots, 50,52\}$.

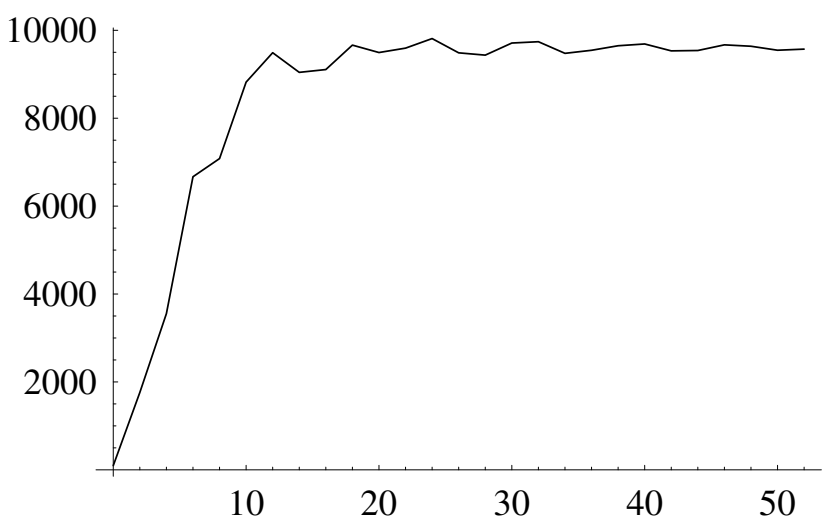

Figure 5. $\nu_{3}^{(N)}(0.50)$ as a function of $N \in\{0,2, \ldots, 50,52\}$.

The 16-digits representation of the coefficients $\nu_{3 j}$ for all $j \in\{0, \ldots, 52\}$ can be obtained from equations (5.12)-(5.26); more precisely,

$$
\nu_{3 j}=\text { coefficient of } t^{j} \text { in }(5.12) \text {, for } j=0, \ldots, 52 \text {. }
$$

From the above data, one can try to make predictions on the convergence radius $\theta_{3}$ of the series $\sum_{j=0}^{+\infty} \nu_{3 j} t^{j}$. In Figures 4-7 we report the partial sums $\nu_{3}^{(N)}(t)$ as functions of $N \in\{0,2, \ldots 50,52\}$, in the four cases $t=0.45,0.50,0.55,0.60$. For $t=0.45$, the function $N \mapsto \nu_{3}^{(N)}(t)$ seems to approach a limit value for large $N$. The situation is not clear for $t=0.50$, due to the appearance of small oscillations; for $t=0.55$ and $t=0.60$, the oscillations of $N \mapsto \nu_{3}^{(N)}(t)$ are large and their amplitude increases with $N$. We regard these results as indicating that $\sum_{j=0}^{+\infty} \nu_{3 j} t^{j}$ is convergent for $t \leqslant 0.45$ and not convergent for $t \geqslant 0.55$; in other words, for the convergence radius we have a conjectural estimate

$$
0.45<\theta_{3}<0.55
$$




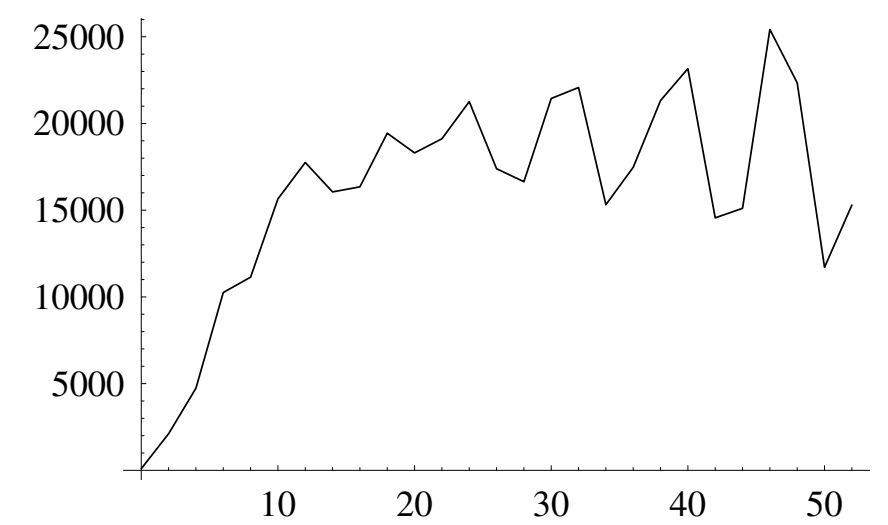

Figure 6. $\nu_{3}^{(N)}(0.55)$ as a function of $N \in\{0,2, \ldots, 50,52\}$.

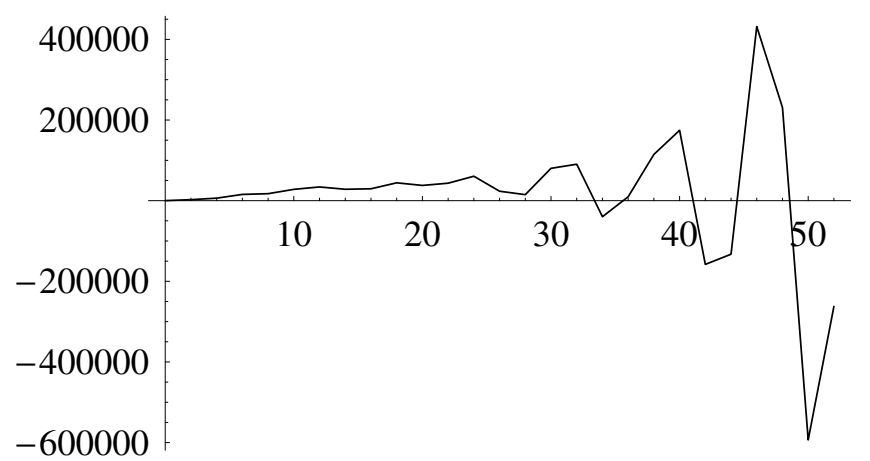

Figure 7. $\nu_{3}^{(N)}(0.60)$ as a function of $N \in\{0,2, \ldots, 50,52\}$.

Another way to estimate $\theta_{3}$ comes from the root test (5.23). Figure 8 is a graph of $\left|\nu_{3 j}\right|^{-1 / j}$ as a function of $j \in\{2,4, \ldots, 50,52\}$. For $j=36,38, \ldots, 52$, there is a fairly good interpolation

$$
\left|\nu_{3 j}\right|^{-1 / j} \simeq 0.484-\left(\frac{8.48}{j}\right)^{2.19}
$$

(obtained assuming for the interpolant the form $A-(B / j)^{c}$, and applying the least squares criterion); here, the right-hand side approximates $\left|\nu_{3 j}\right|^{-1 / j}$ with a mean quadratic error $<0.01$ (let us repeat it, for $j$ between 36 and 52). Assuming that the above interpolant behaves similarly for all larger (even) $j$, and considering $\theta_{3}=\liminf _{j \rightarrow+\infty}\left|\nu_{3 j}\right|^{-1 / j}$ we are led to use $0.484 \pm 0.01$ as upper and lower bounds for it; rounding up to two digits we obtain the inequality

$$
0.47<\theta_{3}<0.50
$$

which is compatible with (5.29). Now, recalling that $\theta_{3}$ is a lower bound on the time of existence $T$ of the solution $u$ (see (5.24)), we are led to the final estimate

$$
0.47<T \leqslant+\infty .
$$

In particular, as anticipated, we have indications that $u$ does not blow up near the $H^{3}$-convergence radius $\tau_{3}$. 


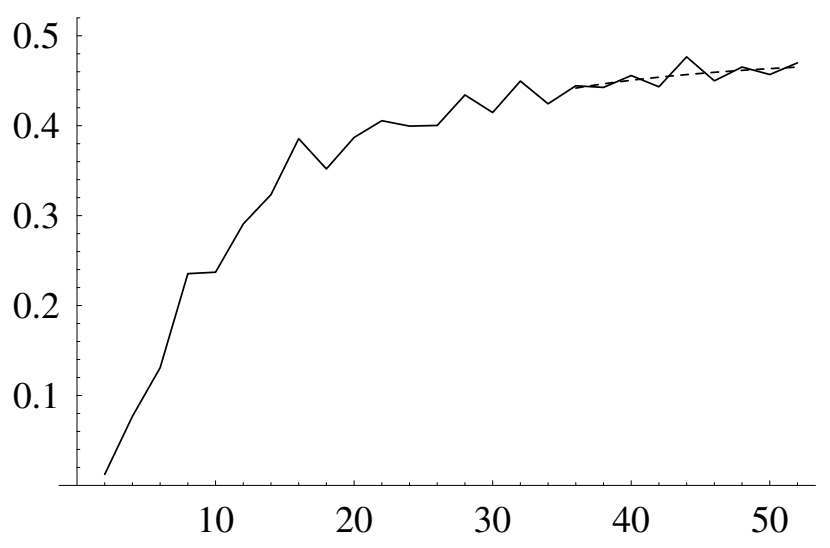

Figure 8. $\left|\nu_{j}\right|_{3}^{-1 / j}$ as a function of $j \in\{2,4, \ldots, 50,52\}$. The dashed line is the graph of the interpolant in (5.30), for $j \in[36,52]$.

\section{Possible Blow-up at larger times for the Behr-NeČas-Wu datum, Via PADÉ APPROXIMANTS}

A few words on Padé approximants. Let us be given an analytic function $f: I \rightarrow \mathbf{C}, t \mapsto f(t)$, with $I$ a neighborhood of zero in $\mathbf{R}$ or $\mathbf{C}$. Let $p, q \in\{0,1,2, \ldots\}$; we recall that the Padé approximant of order $(p, q)$ of $f$, if it exists, is the unique complex function $[p / q]_{f} \equiv[p / q]$ of the form

$$
[p / q](t)=\frac{a_{0}+a_{1} t+\ldots+a_{p} t^{p}}{1+b_{1} t+\ldots b_{q} t^{q}}
$$

such that

$$
f(t)=[p / q](t)+O\left(t^{p+q+1}\right) \text { for } t \rightarrow 0 .
$$

The above condition determines the $p+q+1$ unknown coefficients $a_{0}, \ldots, b_{q}$ as functions of the derivatives $f^{(j)}(0), j=0, \ldots, p+q$; the domain of $[p / q]$ is the largest subset of $\mathbf{C}$ where the above ratio is defined. The family of all approximants $[p / q](p, q=0,1,2, \ldots)$ forms the so-called Padé table of $f$; the approximants with $p=q$ are called diagonal (and the term "near-diagonal" is used if $p \simeq q$ ).

There are several results and conjectures about the convergence to $f$ of the Padé approximants $[p / q]$ with $p$ or $p, q$ large. In particular, the so-called "Padé conjecture" (or "Baker-Gammel-Wills conjecture") states that, for a meromorphic function $f$ on a disk of $\mathbf{C}$, there is a subsequence $\left[p_{\ell} / p_{\ell}\right](\ell=1,2,3, \ldots)$ of diagonal Padé approximants that, for $\ell \rightarrow+\infty$, converges to $f$ uniformly on each compact subset of the disk minus the poles of $f$. This conjecture has been proved for special classes of meromorphic functions (see $[1,23,24]$ and references therein).

It is found experimentally that the Padé approximants of large order work as well for many non meromorphic functions, describing accurately their behavior even close to non polar singularities. This is found to happen in many cases for the diagonal approximants $[p / p]$ (as well as for the near-diagonal ones).

Padé approximants for $\|\boldsymbol{u}(\boldsymbol{t})\|_{3}^{2}$, and possible evidence for a blow-up. The previous considerations can be applied (for suitable $n$ ) to the function $f(t):=\|u(t)\|_{n}^{2}$, where $u$ is the solution of the Euler equation with a given datum $u_{0}$. 
One can ascribe to a number of works the idea of using the Padé approximants for such a function; as in the Introduction, we mention $[9,13,17,22]$ (and some references therein). As already remarked, these papers have considered initial data $u_{0}$ different from the one of Behr-Nečas-Wu (e.g., the Taylor-Green vortex); furthermore, they have generally considered the Sobolev norm of order $n=1$.

Here we are focusing on the (maximal $\mathcal{A}$-) solution $u$ for the Behr-Nečas-Wu datum; from now on, $[p / q]$ stands for the Padé approximant of order $(p, q)$ of the analytic function

$$
t \mapsto f(t):=\|u(t)\|_{3}^{2} .
$$

We conjecture that the diagonal or near-diagonal Padé of sufficiently large order approximate well the function $f$ (and its analytic continuation to the complex plane). From the previous paragraphs, we have the derivatives $f^{(j)}(0)=j ! \nu_{3 j}$ for $j=0, \ldots, 52$; this information allows to determine all the Padé approximants $[p / q]$ for $p+q \leqslant 52$ (and, in particular, all the diagonal approximants $[p / p]$ for $p \leqslant 26$ ). Since $f$ is an even function of $t$, one can restrict the attention to cases with $p$ and $q$ even $\left({ }^{11}\right)$.

Let us consider, for example, the approximant [12/12]. This is found to exist; its numerator and denominator are polynomials with rational coefficients, too large to be written explicitly, but we can use the 16-digits approximation for the coefficients and write

$$
\begin{gathered}
{[12 / 12](t)=\frac{N_{12}(t)}{D_{12}(t)}} \\
N_{12}(t):=96+6.680481407149543 \times 10^{3} t^{2}+3.08095009988031 \times 10^{4} t^{4} \\
+2.3462351635051233 \times 10^{5} t^{6}+2.407391215430808 \times 10^{5} t^{8} \\
+2.5575522886490226 \times 10^{6} t^{10}+3.094974424148063 \times 10^{6} t^{12}, \\
D_{12}(t):=1+0.255014657807743 t^{2}+4.288322833232482 t^{4}-5.985294148961588 t^{6} \\
+8.973150435320479 t^{8}+66.29326162173366 t^{10}-612.1107629833056 t^{12} .
\end{gathered}
$$

The poles of [12/12], which are the zeros of $D_{12}$, are simple and occur at the points

$$
\begin{gathered}
t= \pm 0.294020 \pm 0.464361 i \quad(|t|=0.549617) ; \\
t= \pm 0.511609 \pm 0.301416 i \quad(|t|=0.593797) ; \\
t= \pm 0.606004 i, \quad t= \pm 0.626199
\end{gathered}
$$

(here and in the sequel, \pm means that we can choose independently the signs for the real and imaginary part, e.g.,+ for the real and - for the imaginary part). So, the singularities of minimum modulus of the approximant $[12 / 12]$ are at anyone of the points $T_{\circ}= \pm 0.294020 \pm 0.464361 i$, such that $\left|T_{\circ}\right|=0.549617$; furthermore, the real singularities closest to the origin are at anyone of the points $T_{*}= \pm 0.626199$.

We have performed a similar analysis for all the approximants $[p / p]$, with $p=14,16, \ldots, 26$ and for some near-diagonal approximants $[p / q]$, with $p+q=50$ or 52 ; the results are summarized in Table 2 .

Let us point out some features of the Padé approximants in Table 2, with their possible implications.

(i) For all the approximants, the poles of minimum modulus occur at points $T_{\circ}$ with $\left|T_{\circ}\right| \simeq 0.5$. It is difficult to extrapolate a limit value of $\left|T_{\circ}\right|$, so we limit ourselves to consider the mean $\left\langle\left|T_{\circ}\right|\right\rangle$ and the mean quadratic error $\Delta\left|T_{\circ}\right|$ over some sample values of $(p, q)$ extracted from Table 2; these are reported in Table 3, where

\footnotetext{
${ }^{11}$ In a neighborhood of zero $f$ has a series expansion in even powers of $t$, so it has the form $f(t)=F\left(t^{2}\right)$ for a suitable function $s \mapsto F(s)$. In any situation of this type, writing [/ ] and [/ $]_{F}$ for the Padé approximants of $f$ and $F$ as functions of $t$ and $s$, respectively, we have the following: for $k, m \in \mathbf{N},[2 k / 2 m](t)=[2 k+1 / 2 m](t)=[2 k / 2 m+1](t)=[k / m]_{F}\left(t^{2}\right)$, provided that $[k / m]_{F}$ exists. Existence of the (odd, odd) approximants of $f$ cannot be granted analyzing the approximants of $F$. (To avoid misunderstandings, we repeat that existence of the Padé approximant of any order $(p, q)$ means that both Eqs. (6.1), (6.2) are fulfilled).
} 
TABLE 2. Poles of some diagonal or near-diagonal Padé approximants $[p / q](t)$ to $\|u(t)\|_{3}^{2} . T_{\circ}:=$ pole closest to the origin (with modulus $\left.\left|T_{\circ}\right|\right) ; T_{*}:=$ real (or almost real) pole closest to the origin.

\begin{tabular}{|l|l|l|l|}
\hline$[p / q]$ & $T_{\circ}$ & $\left|T_{\circ}\right|$ & $T_{*}$ \\
\hline$[12 / 12]$ & $\pm 0.294020 \pm 0.464361 i$ & 0.549617 & \pm 0.626199 \\
{$[14 / 14]$} & $\pm 0.281333 \pm 0.445002 i$ & 0.526474 & \pm 0.656185 \\
{$[16 / 16]$} & $\pm 0.283300 \pm 0.446498 i$ & 0.528790 & \pm 0.661087 \\
{$[18 / 18]$} & $\pm 0.283081 \pm 0.445859 i$, & 0.528134 & \pm 0.660118 \\
{$[20 / 20]$} & $\pm 0.345307 \pm 0.348713 i$ & 0.490752 & $\pm 0.621387 \pm 0.047708 i$ \\
{$[22 / 22]$} & $\pm 0.350239 \pm 0.350695 i$ & 0.495635 & \pm 0.541967 \\
{$[24 / 24]$} & $\pm 0.349063 \pm 0.350777 i$ & 0.494863 & $\pm 0.609804 \pm 0.0383530 i$ \\
{$[26 / 26]$} & $\pm 0.0714399 \pm 0.508700 i$ & 0.513692 & \pm 0.816133 \\
\hline$[22 / 28]$ & $\pm 0.0278472 \pm 0.462259 i$ & 0.463097 & $\pm 0.658688 \pm 0.0671444 i$ \\
{$[28 / 22]$} & $\pm 0.0726664 \pm 0.513427 i$ & 0.518544 & $\pm 0.854937 \pm 0.0798538 i$ \\
{$[24 / 26]$} & $\pm 0.0275015 \pm 0.462148 i$ & 0.462965 & $\pm 0.658586 \pm 0.0671042 i$ \\
{$[26 / 24]$} & $\pm 0.0714492 \pm 0.506794 i$ & 0.511806 & $\pm 0.769049 \pm 0.0923157 i$ \\
{$[22 / 30]$} & $\pm 0.110906 \pm 0.503728 i$ & 0.515792 & \pm 0.577969 \\
{$[30 / 22]$} & $\pm 0.0723087 \pm 0.511164 i$ & 0.516253 & $\pm 0.887455 \pm 0.0401287 i$ \\
{$[24 / 28]$} & $\pm 0.0131675 \pm 0.457291 i$ & 0.457481 & $\pm 0.660114 \pm 0.0673984 i$ \\
{$[28 / 24]$} & $\pm 0.0716786 \pm 0.509393 i$ & 0.514411 & $\pm 0.807680 \pm 0.0925495 i$ \\
\hline
\end{tabular}

TABLE 3. Means and mean quadratic errors of $\left|T_{\circ}\right|$ and $\left|T_{*}\right|$, averaging over three sets $S_{1}, S_{2}, S_{3}$ of pairs $(p, q)$ from Table 2. $S_{1}:=\{(p, p) \mid p$ even, $12 \leqslant p \leqslant 26\} ; \quad S_{2}:=$ $\{(p, p) \mid p$ even, $20 \leqslant p \leqslant 26\} ; S_{3}:=\{(p, q)$ in Table $2 \mid 50 \leqslant p+q \leqslant 52\}$.

\begin{tabular}{|l|l|l|l|l|}
\hline Set & $\left\langle\left|T_{\circ}\right|\right\rangle$ & $\Delta\left|T_{\circ}\right|$ & $\left\langle\left|T_{*}\right|\right\rangle$ & $\Delta\left|T_{*}\right|$ \\
\hline$S_{1}$ & 0.515995 & 0.0195414 & 0.649489 & 0.072747 \\
$S_{2}$ & 0.498735 & 0.00883212 & 0.648081 & 0.101851 \\
$S_{3}$ & 0.497116 & 0.0255134 & 0.746256 & 0.101273 \\
\hline
\end{tabular}

we average over three distinct sets $S_{1}, S_{2}$ and $S_{3}$ of pairs $(p, q)$ (the first two made of diagonal pairs, the third one containing also near-diagonal pairs).

For a holomorphic function, the convergence radius of the power series centered at zero is the modulus of the singularity closest to the origin. So, assuming that the above $[p / q]$ Padè describe approximately the singularities of $f(t)=\|u(t)\|_{3}^{2}$, one can derive from them an estimate of the convergence radius $\theta_{3}$ for the power series of $f(t)$. For example, one could assume $\left|T_{\circ}\right|-\Delta\left|T_{\circ}\right|<\theta_{3}<\left|T_{\circ}\right|+\Delta T_{\circ}$, where the mean and the mean quadratic error are taken over a suitable set of approximants. Using the sets $S_{1}, S_{2}, S_{3}$ of Table 3 (and rounding $\left|T_{\circ}\right| \pm \Delta\left|T_{\circ}\right|$ to 2 digits) one obtains, respectively,

$$
0.49<\theta_{3}<0.54, \quad 0.48<\theta_{3}<0.51, \quad 0.47<\theta_{3}<0.53
$$

anyone of these estimates has a significant overlap with equation (5.31), that we still regard as our basic estimate on $\theta_{3}$.

(ii) The $[p / q]$ approximants of Table 2 have real poles or "almost real" poles, close to the real axis; real poles occur more frequently in the diagonal cases $\left({ }^{12}\right)$. For all the $[p / q]$ approximants in Table 2 , we have denoted with $T_{*}$ the real (or almost real) singularities closest to the origin. One notes rather large oscillations of $\left|T_{*}\right|$ as a function of the order, even for the largest available values of $p, q$. In Table 3 we report the mean

\footnotetext{
${ }^{12}$ The occurrence of almost real singularities has also been pointed out in $[13,22]$ while analyzing the Padé approximants for $\|u(t)\|_{1}^{2}$, with initial conditions $u_{0}$ different from the Behr-Nečas-Wu datum.
} 
$\left\langle\left|T_{*}\right|\right\rangle$ and the (rather large) mean quadratic error $\Delta\left|T_{*}\right|$, obtained averaging over the sets of approximants $S_{1}, S_{2}$ and $S_{3}$.

The above results on the singularities $T_{*}$ somehow suggest that $f(t)=\|u(t)\|_{3}^{2}$ could diverge for $t \rightarrow T^{-}$ (and $t \rightarrow(-T)^{+}$), for a suitable $T$. One could assume the upper and lower bounds $\left\langle\left|T_{*}\right|\right\rangle-\Delta\left|T_{*}\right|<T<$ $\left\langle\left|T_{*}\right|\right\rangle+\Delta\left|T_{*}\right|$, where the means are taken over anyone of the sets in Table 3 ; averaging over $S_{1}, S_{2}, S_{3}$ one obtains, respectively,

$$
0.57<T<0.73, \quad 0.54<T<0.75, \quad 0.64<T<0.85 .
$$

If the conjectured divergence of $f(t)$ actually occurred, the solution $u$ of the Euler equation with the BehrNečas-Wu datum would blow up at $T$ (and $-T$ ); admittedly, the indications for such a blow-up are weak.

D-log Padè approximants for $\|\boldsymbol{u}(\boldsymbol{t})\|_{3}^{2}$. As well known, the D-log Padé approximants of a function $t \mapsto f(t)$ (with $f(0) \neq 0$ ) are the Padé approximants for the logarithmic derivative $\dot{f} / f(\cdot:=\mathrm{d} / \mathrm{d} t)$. These approximants are generally regarded as more suitable for describing the behavior of $f$ close to singularities, even of non polar type. In particular, the presence of a singularity at a point $T_{*}$, say real, and a behavior of the type $[p / q]_{\dot{f} / f} \sim \lambda_{*} /\left(T_{*}-t\right)$ for $t \rightarrow T_{*}^{-}$is regarded as an indication that $f(t) \sim$ const $/(T-t)^{\lambda}$ for real $t \rightarrow T^{-}$, where $T \simeq T_{*}$ and $\lambda \simeq \lambda_{*}[1]$.

The function $f(t):=\|u(t)\|_{3}^{2}$ is even in $t$, so it is natural to consider its (odd,odd) D-log approximants $\left({ }^{13}\right)$. More precisely, we have considered the diagonal approximants $[p / p]_{\dot{f} / f}$, with odd $p \leqslant 25$; the results are very unstable with respect to the order, and ultimately not sufficient to get any indication of blow-up $\left({ }^{14}\right)$.

\section{Conclusions}

The previous results about the Behr-Nečas-Wu datum $u_{0}$ support our statements in the introduction, i.e.:

(a) The power series for $u_{0}$ has an $H^{3}$ convergence radius $\tau_{3}$ such that $0.32<\tau_{3}<0.33$ (see Eq. (5.15)).

(b) There is no blow-up at $\tau_{3}$ and the (maximal $\mathcal{A}$-) solution $u$ of the Euler Cauchy problem exists, at least, up to a time $\theta_{3}$ (the convergence radius for the series expansion of $\left.\|u(t)\|_{3}^{2}\right)$, for which we have from $(5.31$ ) the estimate $\theta_{3}>0.47$.

(c) The Padé approximants for $\|u(t)\|_{3}^{2}$ in Table 2 give weak indications that $u$ might blow up at a time $T$, with $0.54<T<0.85$ (see Eq. (6.7)).

We think that the evidence given in this paper is rather strong for (a)(b). As for (c), doubts on the blow-up conjecture arise not only from the rather erratic behavior of the real (and almost real) singularities in the computed Padé approximants; in fact there are more general reasons, recalled at the end of the Introduction, suggesting caution in deriving blow-up results from Padé analysis.

Acknowledgements. We are grateful to Paolo Butera and to the anonymous referees for useful bibliographical indications and for suggestions about the style of the paper and the use of Padé approximants. Furthermore, we acknowledge one of

\footnotetext{
${ }^{13}$ We have $f(t)=F\left(t^{2}\right)$, for a suitable function $s \mapsto F(s)$; in any case like this, for $k, m \in \mathbf{N}$ one finds $[2 k+1 / 2 m+1]_{\dot{f} / f}(t)=$ $[2 k+2 / 2 m]_{\dot{f} / f}(t)=[2 k+1 / 2 m]_{\dot{f} / f}(t)=2 t[k / m]_{F^{\prime} / F}\left(t^{2}\right)$, provided that $[k / m]_{F^{\prime} / F}$ exists (of course ${ }^{\prime}:=d / d s$, and we are considerind the D-log approximants of $F$ as a function of $s$ ). Existence of the (even, odd) D-log approximants of $f$ cannot be granted analyzing the D-log approximants of $F$.

${ }^{14}$ Here is a more precise description of the computational outcomes. The D-log approximants of order $(p, p)$ for $p=17,19,21$ have real singularities at points $T_{*} \simeq 0.72$ and are such that $[p / p]_{\dot{f} / f} \sim \lambda_{*} /\left(T_{*}-t\right)$ for $t \mapsto T_{*}^{-}$, with $\lambda_{*} \simeq 2.6$; so, for $\|u(t)\|_{3}=\sqrt{f(t)}$ we have a conjecture $\|u(t)\|_{3} \sim$ const./ $(T-t)^{\alpha}$ with $T \simeq 0.72$ and $\alpha \simeq \lambda_{*} / 2 \simeq 1.3$. This value of $\alpha$ agrees with the theoretical bound $\alpha \geqslant 1$ in the event of blow-up (see Eq. (2.16)); it agrees as well with the (conjectural) bound $\alpha \geqslant 6 / 5$, obtained extrapolating from $\mathbf{R}^{3}$ to $\mathbf{T}^{3}$ the estimate $(2.17)$. On the contrary, the D-log approximant of order $(23,23)$ for $f$ has no real (nor almost real) singularity. Finally, at the order $(25,25)$ there is a real singularity for $T_{*} \simeq 0.52$, and $[25 / 25]_{\dot{f} / f} \sim \lambda_{*} /\left(T_{*}-t\right)$ for $t \mapsto T_{*}^{-}$, with $-0.002<\lambda_{*}<0.002$ (there are numerical difficulties in a more precise determination of $\lambda_{*}$ ). Returning to $\|u(t)\|_{3}=\sqrt{f(t)}$, the $[25,25]$ Padé would suggest $\|u(t)\|_{3} \sim$ const. $/(T-t)^{\alpha}$ with $T \simeq 0.52$ and $-0.001 \lesssim \alpha \lesssim 0.001$. This statement is an absurdity even in the case $0<\alpha \lesssim 0.001$, since it contradicts the bound $(2.16) \alpha \geqslant 1$.
} 
the referees for a very useful remark on how to derive Proposition 2.3 from the existing literature on analytic solutions of the Euler equation. This work was supported by INdAM, INFN and by MIUR, PRIN 2008 Research Project "Geometrical methods in the theory of nonlinear waves and applications".

\section{REFERENCES}

[1] G.A. Baker and P. Graves-Morris, Padé approximants, 2nd edition, Cambridge University Press, Cambridge. Encycl. Math. Appl. 59 (1996).

[2] M.S. Baouendi and C. Goulaouic, Sharp estimates for analytic pseudodifferential operators and application to Cauchy problems. J. Differ. Equ. 48 (1983) 241-268.

[3] C. Bardos and S. Benachour, Domaine d'analycité des solutions de l'équation d'Euler dans un ouvert de $\mathbf{R}^{n}$. Annal. Scuola Norm. Sup. Pisa Cl. Sci. 4 (1977) 647-687.

[4] C. Bardos and E.S. Titi, Euler equations for incompressible ideal fluids. Russian Math. Surveys 62 (2007) $409-451$.

[5] J. T.Beale, T. Kato and A. Majda, Remarks on the breakdown of smooth solutions for the 3D Euler equations. Commun. Math. Phys. 94 (1984) 61-66.

[6] E. Behr, J. Nečas and H. Wu, On blow-up of solution for Euler equations. ESAIM: M2AN 35 (2001) $229-238$.

[7] N. Bourbaki, Éléments de Mathématique. Variétés différentielles et analytiques, Fascicule de résultats, Hermann, Paris (1971).

[8] M.E. Brachet, D. Meiron, S. Orszag, B. Nickel, R. Morf and U. Frisch, Small scale structure of the Taylor-Green vortex. J. Fluid Mech. 130 (1983) 411-452.

[9] M.E. Brachet, D. Meiron, S. Orszag, B. Nickel, R. Morf and U. Frisch, The Taylor-Green vortex and fully developed turbulence. J. Statist. Phys. 34 (1984) 1049-1063.

[10] M.E. Brachet, M. Meneguzzi, A. Vincent, H. Politano and P.L. Sulem, Numerical evidence of smooth self-similar dynamics and possibility of subsequent collapse for three-dimensional ideal flows. Phys. Fluids A 4 (1992) 2845-2854.

[11] T. Chen and N. Pavlović, A lower bound on blowup rates for the 3D incompressible Euler equation and a single exponential Beale-Kato-Majda estimate completer. ArXiv:1107.0435v1 [math.AP] (2011).

[12] S.I. Chernyshenko, P. Constantin, J.C. Robinson and E.S. Titi, A posteriori regularity of the three-dimensional NavierStokes equations from numerical computations. J. Math. Phys. 48 (2007) 065-204.

[13] U. Frisch, Fully developed turbulence and singularities, in Chaotic Behavior of Deterministic Systems, edited by G. Iooss, R.H.G. Helleman, R. Stora. LesHouches, session XXXVI, North-Holland, Amsterdam (1983) 665-704.

[14] U. Frisch, T. Matsumoto and J. Bec, Singularities of the Euler flow? Not out of the blue!. J. Stat. Phys. 113 (2003) $761-781$.

[15] T. Kato, Quasi-linear equations of evolution, with applications to partial differential equations, in Spectral theory and differential equations, Proceedings of the Dundee Symposium. Lect. Notes Math. 448 (1975) 23-70.

[16] S. Kida, Three-dimensional periodic flows with high-symmetry. J. Phys. Soc. Japan 54 (1985) 2132-2140.

[17] R.H. Morf, S.A. Orszag and U. Frisch, Spontaneous singularity in three-dimensional inviscid, incompressible flow. Phys. Rev. Lett. 44 (1980) 572-574.

[18] M. Morimoto, Analytic functionals on the sphere. AMS, Providence. Transl. Math. Monogr. 178 (1998).

[19] C. Morosi and L. Pizzocchero, On approximate solutions of semilinear evolution equations II. Generalizations, and applications to Navier-Stokes equations. Rev. Math. Phys. 20 (2008) 625-706.

[20] C. Morosi, L. Pizzocchero, An $H^{1}$ setting for the Navier-Stokes equations: Quantitative estimates. Nonlinear Anal. 74 (2011) 2398-2414.

[21] C. Morosi and L. Pizzocchero, On approximate solutions of the incompressible Euler and Navier-Stokes equations. Nonlinear Anal. 75 (2012) 2209-2235.

[22] R.B. Pelz, Extended series analysis of full octahedral flow: numerical evidence for hydrodynamic blowup. Fluid Dyn. Res. 33 (2003) 207-221.

[23] H. Stahl, The convergence of diagonal Padé approximants and the Padé conjecture. J. Comput. Appl. Math. 86 (1997) $287-296$.

[24] S.P. Suetin, Padé approximants and efficient analytic continuation of a power series. Russian Math. Surveys 57 (2002) $43-141$.

[25] F. Treves, Topological vector spaces, distributions and kernels. Academic Press, New York (1967).

[26] GMPY Collaboration, Multiprecision arithmetic for Python, http://code.google.com/p/gmpy. This software is a wrapper for GMP Multiple Precision Arithmetic Library, see http://gmplib.org. 